\title{
Approach to In Situ Component Level Electronics Assembly Repair (CLEAR) for Constellation
}

Peter M. Struk and Richard C. Oeftering

Glenn Research Center, Cleveland, Ohio 


\section{NASA STI Program . . . in Profile}

Since its founding, NASA has been dedicated to the advancement of aeronautics and space science. The NASA Scientific and Technical Information (STI) program plays a key part in helping NASA maintain this important role.

The NASA STI Program operates under the auspices of the Agency Chief Information Officer. It collects, organizes, provides for archiving, and disseminates NASA's STI. The NASA STI program provides access to the NASA Aeronautics and Space Database and its public interface, the NASA Technical Reports Server, thus providing one of the largest collections of aeronautical and space science STI in the world. Results are published in both non-NASA channels and by NASA in the NASA STI Report Series, which includes the following report types:

- TECHNICAL PUBLICATION. Reports of completed research or a major significant phase of research that present the results of NASA programs and include extensive data or theoretical analysis. Includes compilations of significant scientific and technical data and information deemed to be of continuing reference value. NASA counterpart of peer-reviewed formal professional papers but has less stringent limitations on manuscript length and extent of graphic presentations.

- TECHNICAL MEMORANDUM. Scientific and technical findings that are preliminary or of specialized interest, e.g., quick release reports, working papers, and bibliographies that contain minimal annotation. Does not contain extensive analysis.

- CONTRACTOR REPORT. Scientific and technical findings by NASA-sponsored contractors and grantees.
- CONFERENCE PUBLICATION. Collected papers from scientific and technical conferences, symposia, seminars, or other meetings sponsored or cosponsored by NASA.

- SPECIAL PUBLICATION. Scientific, technical, or historical information from NASA programs, projects, and missions, often concerned with subjects having substantial public interest.

- TECHNICAL TRANSLATION. Englishlanguage translations of foreign scientific and technical material pertinent to NASA's mission.

Specialized services also include creating custom thesauri, building customized databases, organizing and publishing research results.

For more information about the NASA STI program, see the following:

- Access the NASA STI program home page at http://www.sti.nasa.gov

- E-mail your question via the Internet to help@ sti.nasa.gov

- Fax your question to the NASA STI Help Desk at $443-757-5803$

- Telephone the NASA STI Help Desk at 443-757-5802

- Write to: NASA Center for AeroSpace Information (CASI) 7115 Standard Drive Hanover, MD 21076-1320 


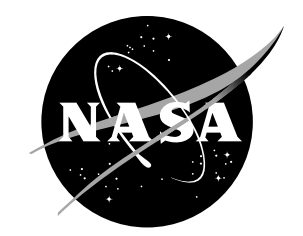

\section{Approach to In Situ Component Level Electronics Assembly Repair (CLEAR) for Constellation}

Peter M. Struk and Richard C. Oeftering

Glenn Research Center, Cleveland, Ohio

Prepared for the

Space 2009 Conference and Exposition

sponsored by the American Institute of Aeronautics and Astronautics

Pasadena, California, September 14-17, 2009

National Aeronautics and

Space Administration

Glenn Research Center

Cleveland, Ohio 44135 


\section{Acknowledgments}

The authors wish to acknowledge the following individuals for their contribution to this work: Eric. E. Anderson, Martin A. Bradish, John W. Easton, Gregory P. Funk, Gary E. Gorecki, Alain Izadnegahdar, Jeffery R. Juergens, Michael J. Lewis, Duc K. Truong, Daniel R. Vrnak, and Raymond P. Wade. The authors acknowledge the contribution of various other NASA organizations including the Logistics and Maintenance group at Johnson Space Center, the NASA Spacecraft Services Depot and NASA Shuttle Logistics Depot. Also, This work was funded by the Supportability Project in NASA's Exploration Technology Development Program Office (Mr. Barmac Taleghani, Program Manager, NASA Langley Research Center).

Trade names and trademarks are used in this report for identification only. Their usage does not constitute an official endorsement, either expressed or implied, by the National Aeronautics and Space Administration.

Level of Review: This material has been technically reviewed by technical management.

Available from

NASA Center for Aerospace Information 7115 Standard Drive

Hanover, MD 21076-1320
National Technical Information Service 5301 Shawnee Road Alexandria, VA 22312 


\title{
Approach to In Situ Component Level Electronics Assembly Repair (CLEAR) for Constellation
}

\author{
Peter M. Struk and Richard C. Oeftering \\ National Aeronautics and Space Administration \\ Glenn Research Center \\ Cleveland, Ohio 44135
}

\begin{abstract}
NASA's Constellation Program for exploration of the Moon and Mars places human crews in extreme isolation and resource scarce environments. Maintenance resupply is a significant issue for such long duration missions. Currently, the International Space Station (ISS) approaches maintenance primarily around replaceable modules called Orbital Replacement Units (ORU). The process of swapping out ORUs has served the ISS well and kept crew time for maintenance to a minimum. However, this approach assumes a substantial logistics capacity to provide replacement ORUs and return ORUs to Earth for repair and return to service. The elimination of the Space Shuttle substantially reduces the supply of replacement units and virtually eliminates the return for repairs.

The modular ORUs used for ISS require relatively large blocks of replacement hardware even though the actual failed component may really be several orders of magnitude smaller. Electronic ORUs are typically modular assemblies that may include several internal circuit cards. While being able to access and replace smaller sub-assemblies such as circuit cards is critical, a capability to repair failed electronic hardware at the component level offers the most potential for reduced resupply mass. Furthermore, such repair capabilities help to enable the crew to better respond to unforeseen faults and achieve more resource independence which becomes significantly more important as humans venture further away from Earth. A capability to repair at the component-level within the confines of a spacecraft requires significant planning and investment by NASA beginning early in the design phases of a mission.

The Component Level Electronics Assembly Repair (CLEAR) task was created to explore electronics repair for future manned space missions. From 2006 to 2009, CLEAR was managed under the Supportability project of the Exploration Technology Development Program. This paper describes the activities and accomplishments of CLEAR. These include making a case for component-level electronics repair, examination of current terrestrial repair hardware and potential repair needs. Based on those needs, the CLEAR team proposes an architecture for an in-site repair capability aboard a spacecraft or habitat. The repair architecture, which includes a combination of manual and semi-automated tools, would allow the crew to diagnose, repair, and test electronics down to the component level while minimizing spares payload with minimal crew time and training. Additionally, this paper discusses recent progress toward developing in-space repair capabilities_including two spaceflight experiments—and presents technology concepts which could help enable or benefit the same.
\end{abstract}

\subsection{Introduction}

NASA's plans for long duration missions to the Moon and, especially, to Mars require a much greater degree of self sufficiency on the part of the crew than ever before. Such missions will have greatly reduced logistic support from Earth compared to International Space Station (ISS). Returning to Earth in the event of an emergency may not be an option either, as a lunar return flight could require two to three days, and a Martian return flight will require much longer.

One area of mission support that NASA must plan for is electronic repairs. Although more reliable than predicted, electronic systems aboard the Space Shuttle and ISS have experienced faults leading to the use of backup systems or loss of capability to some degree (Refs. 1 and 2). Therefore, it is likely that crews of long duration space missions will likely encounter an electronics failure at some point during a 
mission. With the design of the Crew Exploration Vehicle (CEV) already well underway and likely influencing the design of future spacecraft and hardware, it is important for NASA to begin examining and designing techniques and tools for crew members to conduct electronics repair during long duration space mission. These considerations include system design (for accessibility, parts type and sizes, and board complexity), repair infrastructure (including diagnostic capabilities, tools, and other needed equipment), and logistics constraints. The decisions on how to approach each of these considerations depends on the overall repair strategy chosen.

The historical approach to electronics repair on manned spaceflight missions, particularly those aboard ISS, has been to replace modular subassemblies called Orbital Replacement Units (ORU's). The astronauts simply replace the malfunctioning ORU with a spare and then return the faulty ORU to Earth via the Space Shuttle. This approach allows for simplicity in the diagnosis of the problem, as the fault only has to be isolated to a given ORU, rather than determining the fault down to the component level. Crew training and experience in performing repairs are minimized. Once on Earth, the faulty ORU is diagnosed, repaired, and then returned to a pool of spares awaiting re-use in space. With this approach, there is a high degree of confidence in the repair, as the ORU can be tested extensively on the ground prior to being relaunched to the ISS.

Conversely, this approach carries with it a severe penalty in terms of the logistics support required (and overall cost to the program). Conceptually, it is easy to imagine the penalty in mass and volume that is levied when an entire ORU is launched to remedy a failure of a single, small component such as a resistor, transistor, or other electrical component. While this approach is expensive, it may be acceptable for low Earth orbit (LEO) missions if resupply arrives on a regular basis (large quantities of spares are not easily stored in orbit). The resupply interval for the ISS can be as short as a month although early mission planners expected more frequent intervals. For future missions beyond low Earth orbit, the interval between resupply is anticipated to be significantly greater. For example, the resupply interval for the planned lunar outpost is just greater than six months.

Alternatives to the ORU replacements are lower-level repairs. Such repairs can include replacement of lower-level units (also called shop-replaceable units or SRUs), for example circuit board-level swapouts. A further level of repair could then be repairing the board itself at the component-level. This could serve to greatly reduce the planned-spare ORU requirements, while providing a capability to handle unforeseen repair contingencies. The need to handle such unexpected events has been illustrated by several events in NASA flight history. The successful conclusion of the Apollo 13 mission, after an explosion severely damaged the service module, highlights the benefits of having the capability to implement on-the-spot repairs. The consequence of failing to allow for off-nominal repairs can be inferred from the loss of the shuttle Columbia; even if the crew had been aware of the problems with the leading edge tiles, they were not equipped to make repairs in-flight. Since that incident, NASA has made considerable efforts to allow for contingency repairs of problems of that nature (Ref. 3).

As part of the NASA Supportability project of the Exploration Technology Development Program, the Component-Level Electronics-Assembly Repair (CLEAR) task was established to examine, and to the extent possible, demonstrate the feasibility of how crew-members could effectively perform electronic repair down to the component level. In this document, the acronym CLEAR is used to refer both to the task (e.g., project team name) as well as the process of component-level electronics assembly repair. The objectives of CLEAR are composed of four primary areas which are the basic elements of the repair process:

1. Diagnose electronics assemblies to identify faulty component(s) with equipment that fits within the mass, volume, and power constraints of spacecraft.

2. Repair electronics down to the component-level in-situ with processes and materials that are safe and compatible with the space environment which allows the crew to make the necessary repair.

3. Evaluate (e.g., test) and determine that the repaired circuit is safe to return to service.

4. Augment the flight crew with knowledge and skills to diagnose faults and perform repairs without expanding crew size. 
These areas are investigated by a combination of trade studies, analysis, ground based testing, reduced gravity aircraft testing, and actual spaceflight testing aboard the ISS in two experiments. This paper summarizes activities under CLEAR from 2006 to 2009 which include several other papers (Refs. 4 to 7) available in the public domain as well as other unpublished reports which receive more emphasis here. Specifically, this paper presents a case for lower-level electronics repair, describes what sort of repairs might be necessary, and provides a conceptual architecture to meet those needs. The architecture is intended for use as part of a future lunar or Martian base but is applicable to the ISS or other space vehicles or habitats intended for long-duration use.

\subsection{The Case for Electronics Repair}

\subsection{Examining the ISS}

For insight into actual space repair needs, an internal study examined the operational experience of the ISS program through the Problem Reporting and Corrective Action (PRACA) database specifically for electrical and electronic system problems. The ISS has higher than predicted reliability yet, as expected, it has persistent problems. A goal of this study was to identify which on-orbit electrical problems could be resolved by having a robust component-level repair capability available (such as that described later in this paper). A further goal was to identify problems that could benefit from the additional diagnostic and test capability that complement a component-level repair capability.

The study indicated that many problems stem from a small set of root causes that also represent distinct component problems. A subset of these recurring problems, listed as "root cause unknown," remained unresolved due to limitations in current telemetry, built-in test capability, and available diagnostics. The study concluded that roughly 42 percent of on-orbit electrical problems on ISS could be addressed with a component-level repair. Furthermore, 63 percent of on-orbit electrical problems on ISS could benefit from additional external diagnostic and test capability. Problems that would not benefit from a component-level repair capability stem from software, operations, or documentation errors as well as single-event upset (SEU) errors which can be cleared by a register refresh or by executing an error correction code. If no permanent damage occurs, then a component repair is not needed. Additionally, lighting problem which require replacement bulbs (i.e. a logistics end-of-life issue) were not considered repairable. Overall, these results indicate that in-situ component-level repair in combination with diagnostic and test capability can be expected to increase system availability and reduce logistics.

\subsection{Life-Cycle Cost and Resupply Mass Impacts of Lower-Level Electronic Repair}

An internal project study examined the impacts to life-cycle cost and resupply mass on maintaining electronic hardware by comparing different approaches for in-situ repair of electronic hardware. The first approach, which was the baseline to compare against, was replacement of ORUs as is currently performed on the ISS. The second is replacement of SRUs or board level swap outs. Currently, the ISS has one ORU — the Multiplexer Demultiplex (MDM) — which allows for in-situ board-level swap out. The third repair strategy was component-level replacement at the board level. The third strategy is similar to the SRU replacement in that SRUs need to be removed from the system. Instead of being discarded as in option 2, the crew repairs the SRUs which are returned to service or designated as functional spares. For both options 2 and 3, it was assumed that the ORUs are designed to allow access to the lower board levels.

This study generated a life-cycle cost model using existing data from ISS Criticality (Crit) 1 hardware (which denotes life or mission critical items) to investigate the impact on life-cycle cost for different repair strategies. The resulting subset of hardware consisted of sixty-seven different types of electronic ORUs representing many ISS support systems whose functions are expected to be represented in the Lunar Outpost such as power, communications, life support, and command and data handling. The data used came from two primary data sources: the ISS Modeling Analysis Data Set and the ISS Logistics Access data file prepared by Robert Shisko from the NASA Jet Propulsion Laboratory. This study was limited to historical data of Crit 1 hardware (highest reliability) due to the availability of data at the SRU 
level for Crit 1 systems. Crit 2 and lower systems do not have the SRU data readily available and would take considerable time to generate for each system. Furthermore, Crit 1 hardware was selected to minimize the crew labor for performing SRU or component level repairs. Some SRUs are common between ORUs allowing spares to consist of a percentage of the operational ORUs (which can be swapped from location to location). Mean Time Between Failure (MTBF) numbers were used to generate expected failures and are the current ISS values that have been updated throughout its lifetime.

For the component-level repair option, a conceptual repair and diagnostic station was developed for use in this study similar to that proposed later in this paper. This system involves a combined semi-automated diagnostic and rework station, supplemented by a manual repair capability which significantly surpasses the current capability on ISS. The system also involves telemetry with ground support personnel so as to assist the crew in performing the diagnostics and repair functions. This study assumes that the technology needed for such a capability is at a Technology Readiness Level (TRL)-6, as defined by NASA (Ref. 8). The development effort to go to TRL 9 is accounted for in this analysis assuming that the technology starts at TRL 6.

The life-cycle cost model shows that ORU-level repair of the ISS hardware used for this study over a $10 \mathrm{yr}$, steady-state, operational period had a total cost impact of \$818M. This includes the initial cost of the ORU, spares, launch, and maintenance. By implementing a capability to conduct SRU level replacement, the initial investment and operating cost was reduced to $\$ 149 \mathrm{M}$ (an 82 percent savings). By implementing SRU and component level repair strategies, these costs were further reduced to $\$ 130 \mathrm{M}$ (an 84 percent savings). All alternatives included the development costs of unique flight support equipment required for maintenance. The model also captured crew time impacts including both EVA and IVA activities over a 10-yr period. Relative to ORU replacement (1833 hr), crew time increased by 28 percent (2340 hr) with SRU-level replacement while component-level repair of the SRUs increased crew time by 51 percent (2767 hr). Note that the EVA time (420 hr) does not change between alternatives because the numbers of external ORUs failures do not change (only the maintenance approach).

An ORU case study was used to determine the impact to mass for board-level and component-level sparing. Based on this information, a conceptual ORU was generated which had twelve operational units (29.16 kg). This ORU was performing a strictly electrical function (power switch). To maintain an operational status, the MTBF data for the ORU indicated two spares would be required for a 180 day resupply interval (4.86 kg). Implementing a board level replacement would require two spare cards $(0.86 \mathrm{~kg}$, or an 82 percent reduction in spares mass). Using the MTBF data for the components used within the system, a spare component kit $(0.20 \mathrm{~kg}$, or a 96 percent reduction in spares mass) could be used to maintain operational status. These results are consistent with the life-cycle cost model which showed a 95 percent reduction in spares mass system wide for the component-repair option. Thus, the component repair option offers the lowest potential sparing mass.

In summary, component level repair offers both cost benefits and mass savings for extended space missions. The results show that once you deviate from ORU replacement to lower-level SRU replacement, component-level repair does not add additional cost but rather saves costs over the life-cycle even when including the development cost of the added flight hardware. The cost and mass saving would be expected to further increase with the inclusion of lower criticality but still important hardware (like science payloads which are critical to the success of a mission). During future missions, the ability to perform repair of electronic systems will be required and repairs below the ORU level offer mission flexibility and enhanced safety with a capability to respond to unanticipated failures of electronic hardware. This includes risk mitigation from design errors and common mode faults due to an increased commonality amongst hardware. Furthermore, the inability to bring back faulty hardware due to severe return mass limitations necessitate the need for in-situ diagnostics to understand why systems have failed. Additionally, the ability to perform component-level repairs improves the ability to operate within maintenance resource limits. Such a repair capability comes at the expense of increased crew time needed. This increase can be minimized by incorporating tele-operations, automation, and a supportability strategy which considers component-level repair during the mission design phase. 


\subsection{Establishing the Capabilities Needed for Electronics Repair}

\subsection{Current Terrestrial Repair Capabilities}

To determine the capabilities needed studies examined current technology, current repair practices and how repairs of flight systems are organized. The following are the three primary aspects of a complete repair process.

\subsubsection{Diagnostic Activities}

Diagnosis involves examination, observing symptoms, and measuring fundamental parameters to, first, characterize the fault, and second, determine the root cause of a malfunction or fault. It may involve a passive circuit in a power-off condition or an active circuit in a power-on condition. Furthermore, there may be a series of probing measurements intended to locate a problem area followed by refined probing to isolate the problem. For diagnostic operations, the emphasis is on measurements that help isolate a specific cause among many possible causes.

\subsubsection{Repair Activities}

Repair is broadly defined as a physical replacement, rework, or remanufacture of a faulty or failed circuit element and restoring full function. Most often it involves replacement of faulty components or the rework of interconnecting hardware such as solder joints, connectors, or the underlying substrate. In most cases, a failed component is replaced. It is rare that the component itself is amenable to repair (e.g., transformers). Thus for clarity, repair activities actually are replacement of parts at the component level.

\subsubsection{Functional Test Activities}

Functional test is intended to verify that a circuit functions properly in accordance with functional requirements for that system. Direct electrical measurements are made to determine if the unit is meeting its design specifications. Verifying function implies operating (or simulating operation) and observing the circuit as it performs its function in its operational environment. Functional testing often follows the assembly sequence where functional tests are performed at the lowest to the highest levels of assembly. Functional tests include built-in tests (BITs) for both software and hardware, physical parameter measurement, and recording observed behavior in nominal and off-nominal operations. Ultimately, a circuit must be tested as part of the actual system (or simulated system). Early during the project, the CLEAR team conducted a survey examining the tools and devices used to conduct component-level diagnostics, test, and repair in terrestrial settings. Diagnostic devices include instruments to measure characteristics of the component or circuit including voltage and resistance measurement, oscilloscopes, and a variety of signal generators and network analyzers. Other key diagnostic devices include power-off testers which apply low voltage signals to a component or node and compare the result to a known good sample. Such information can help determine the location of a fault on a circuit board. Additionally, the survey examined functional testers which emulate external systems at the inputs and outputs of a circuit board to verify proper operation and to isolate faults. Key components of functional test systems are shown in Figure 1.

The tools used for performing the physical repair include a variety of hand and soldering tools, including common resistive soldering irons, hot air devices, infrared soldering, and laser soldering systems. Many of the tools are discussed elsewhere in this paper. The survey also examined semiautomated rework stations. Finally, the study surveyed the types and methods to remove conformal coatings which protect a circuit board. Data was tabulated for mass and volume of existing tools, capabilities, and disadvantages of the tools in a space environment. 


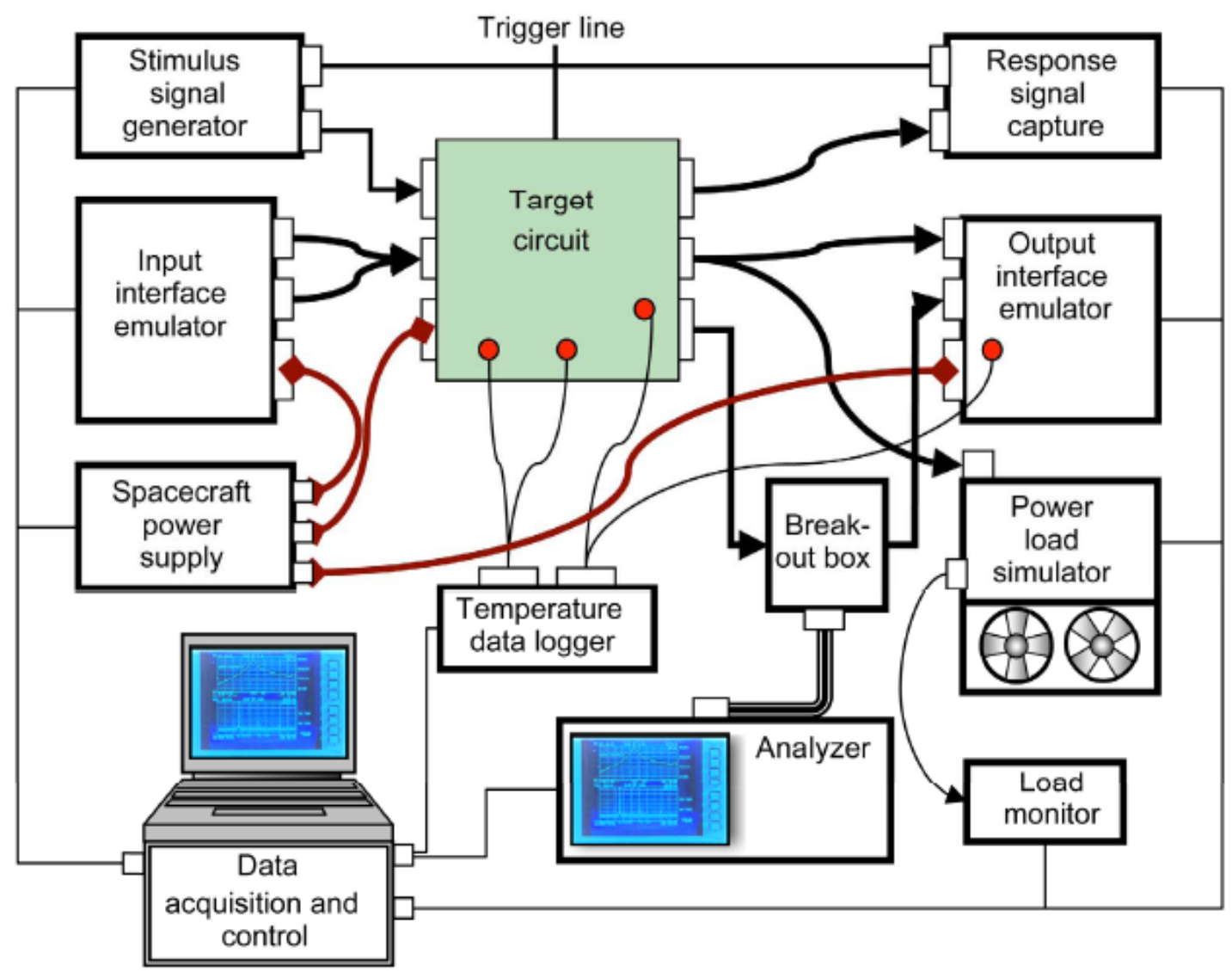

Figure 1.-Conventional functional test systems involve interface emulators, data loggers, signal monitors, signal stimulus sources, measurement instruments, power supplies, and load simulators (with cooling).

\subsection{Current ISS Repair Capabilities}

Current electronics repair strategies on the ISS focus on removing an ORU with faulty electronics and replacing it with a spare unit. With the exception of the MDM discussed previously, current ORUs have not been designed to readily remove circuit cards from higher-level assemblies. Using built-in test (BIT) capabilities and an external test kit, the crew can isolate a fault on an MDM to the circuit card level. The crew then uses a circuit card puller to remove the faulty circuit card. Such a design strategy helps facilitate circuit card removal and is recommended for future designs employing circuit cards. However, the crew does not attempt a component-level repair at the circuit card level.

The ISS crew also has access to some tools suitable for electronics repairs. The crew has access to two soldering irons including one provided by the Russians as well as the United States (U.S.). The Russian iron is a capillary flow type soldering iron which is powered by a constant voltage power source. However, further information on the Russian iron is limited. The U.S. Soldering Kit has a battery powered soldering iron, two Weller PT Series $600^{\circ} \mathrm{F}$ soldering iron tips, a spool of solder wire, solder wick, and heat sinks for performing limited soldering and desoldering tasks. This kit has been used in the past for some repairs (Ref. 9), scientific research (Ref. 10), as well as engineering studies on solder joint formation (Refs. 11 and 12) and manual component-level repair (Ref. 12). These engineering studies were conducted by the CLEAR team and are discussed more in Section 5.0. While useful, this soldering kit is not ideal for repairs of circuit cards at the component level. Some shortcomings include the maximum tip temperature and temperature control methods as well as the limited number of solder tips available. Section 5.4 discusses recommendations to improve soldering capabilities for future space missions. 


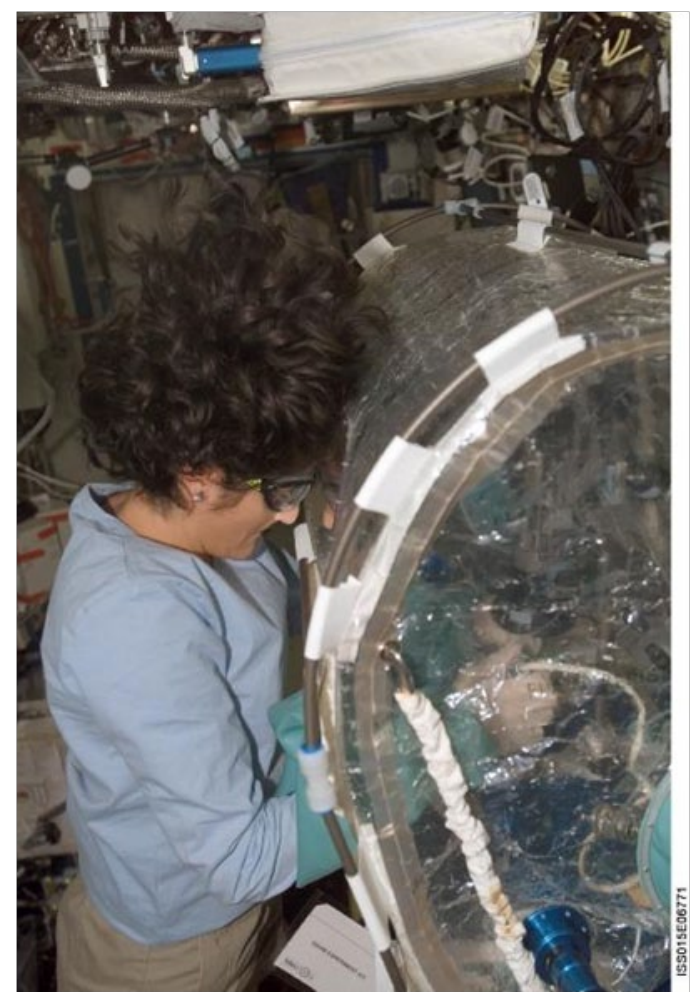

Figure 2.-Astronaut Sunita Williams conducts a soldering experiment within the Maintenance Work Area aboard the ISS.

The crew members conduct soldering tasks within a containment system, a tent-like glovebox with clear plastic sides mounted on the Maintenance Work Area (MWA). Figure 2 shows astronaut Sunita Williams using the MWA with the containment system for soldering aboard the ISS. The containment enclosure traps debris to help prevents contamination of the cabin or life support equipment. The containment system has a small Fresnel lens window that provides a small amount of magnification within the work area. The containment system also includes two feedthroughs, one for a vacuum hose to collect gases and debris evolved from the soldering process, and another to provide power to instruments, such as video cameras, within the work area. The containment system, while effective at containing debris formed during soldering, does have some limitations. The containment system is designed to collapse and fold for storage; performing this operation multiple times has created many creases and folds in the system walls, making it difficult to see through any area but the Fresnel lens. The containment system is also somewhat tall, leading to a large distance (on the order of $12 \mathrm{in}$.) between the work piece and the crew member's eye. This can make seeing the work piece difficult with the naked eye, and limits the effectiveness of a magnifying lens visor provided for the crew because the work piece is beyond the focal length of the lens. This distance between the work piece and closest viewing location can also have ergonomic effects, and become uncomfortable for tall crew members. Finally, the MWA and containment tent take 30 min or more to setup up which has a significant impact on crew time.

Crew members also have access to a wide variety of additional tools as shown in Figure 3. For example, the ISS Pin Kit contains jumper wires with various terminations, connectors, pin, and lugs for making wire connections. The various drawers of the Inner Vehicular Activity (IVA) Tool Kit contains a number of common hand tools, most of which are appropriate for mechanical rather than electronics tasks, but does include crimper tools used with the pins and lugs found in the Pin Kit. The recent Component Repair Experiment (Ref. 12) aboard ISS included a small tool kit augmenting the tools already on orbit. The additional tools included standard and fine tip cutters; three types of tweezers, a dental pick for scraping conformal coating or manipulating small component legs; soldering iron tips appropriate for smaller components; a fiberglass stick for rubbing conformal coating off the circuit board; and liquid flux, flux cored solder wire, and solder wick. These tools remain aboard the ISS and are available for future use. Finally, the crew also has access to some limited diagnostic and testing devices including a combination multimeter and oscilloscope, logic analyzer, power supply, and other basic electronics diagnosis tools, as well as the dedicated MDM tester already discussed.

Repair tasks aboard the ISS depend on extensive guidance from ground support teams. These teams collect data and reports from the crew after a failure, and use this information in an attempt to determine the cause of the fault and a potential solution to the failure. These efforts range from working on fully functional mock-ups of flight instruments and systems on the ground, to communicating and meeting with the developer of the hardware, whether within NASA or an outside vendor. The team then develops and test procedures for a repair, a process that often requires intensive work. The procedures are then transmitted to the crew who, guided by the procedures, attempt to perform a repair and return a faulty system or device to a functional state. 


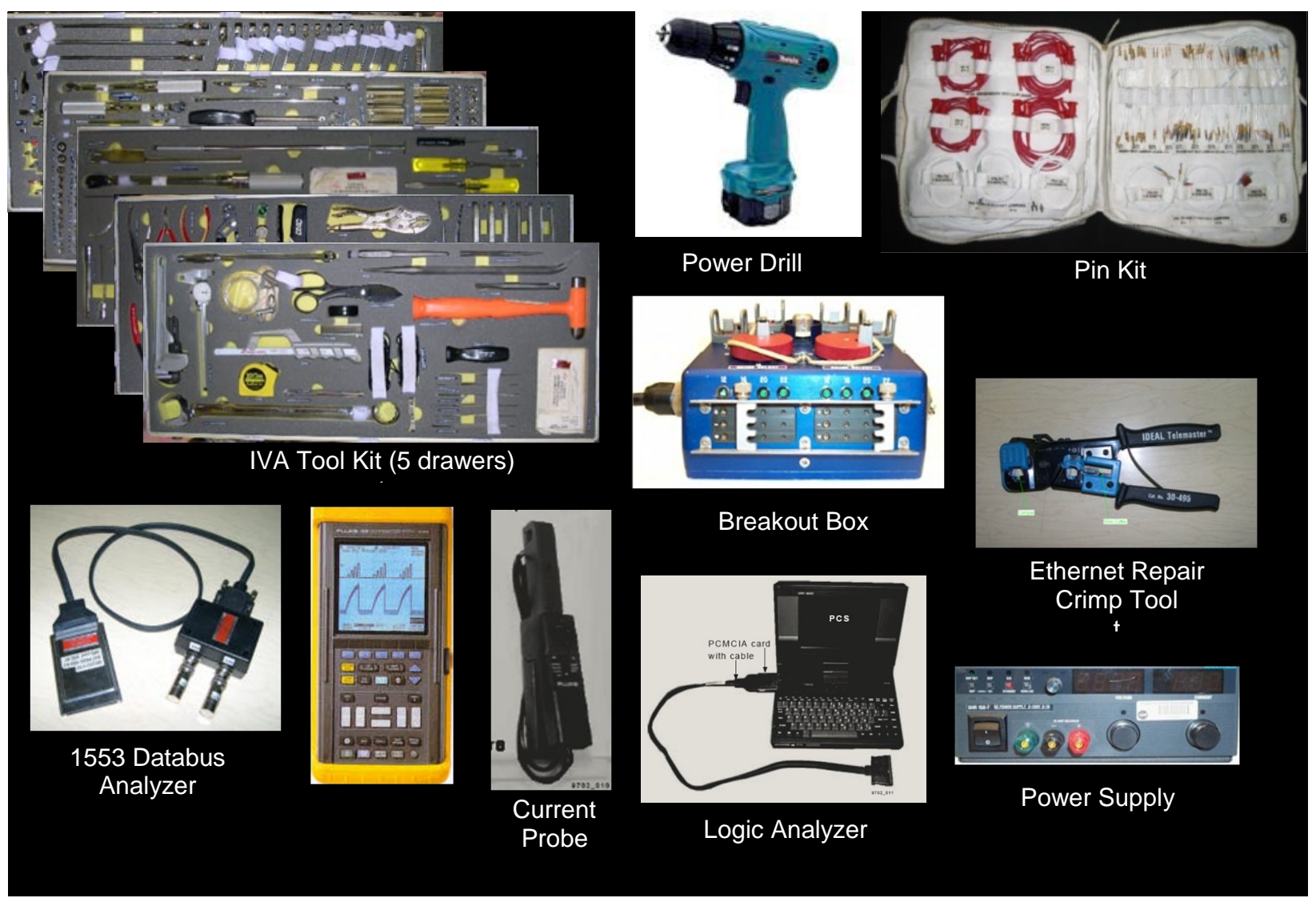

Figure 3.-A sample of tools available aboard the ISS.

\subsection{In-Situ Repair Needs for Electronic Systems of Future Spacecraft}

It is likely that the first operational need for component-level repair capabilities is during longduration outpost stays on the Moon and especially Mars. However, the ISS is a prime location to evaluate such technology and, because of the retirement of the space-shuttle in 2010, may benefit from a repair capability to help sustain systems with reduced logistical support. To better understand what types of electronic components would need to be repaired, the CLEAR team investigated electronic systems to determine the nature of the circuits used during spaceflight. The information was used as a basis to develop an architecture that addresses electronic diagnostics, repair, and testing needs, and the practical constraints of operating in a space environment.

As circuit repair is very dependent on the manufacturing processes, this study relied on data gathered from the ISS Vehicle Master Database (VMDB) for relevant information on design, development, fabrication, and test practices. The rationale for using the ISS is based on the current state of the Constellation Program (CxP) designs and the fact that the ISS is the only predecessor for electronics composed of modular orbital replaceable units (ORUs) — design practices used for CxP will likely be derived from ISS experience. New CxP spacecraft such as the Orion are expected to employ state of the art field programmable devices and high density grid array packaging. Because lunar outpost is over a decade away, advances in electronics at the component level will affect diagnostics, repair, and test technology. The electronics industry however, has shown signs of maturing and the rate of performance increase is slowing. Therefore, electronic equipment designed for the current generation spacecraft are expected to be relevant for lunar applications.

Finally, the study also made some unexpected but important findings regarding information access and the ability to formulate diagnostic, repair, and test solutions for electronic circuits. It was found that too much of the information provided in the ISS VMDB is subject to the discretion of the original vendor. 
The viability of component-level repair depends heavily on rapid access to detailed design information, including the process and materials used in a circuit's construction. Such information accessibility must be built into CxP.

\subsubsection{Diagnostics and Functional Test}

Terrestrially, repairs typically require dedicated equipment to perform initial diagnostics and post repair functional tests. Conventional diagnostics and test equipment for a single unit may require multiple racks, while repair equipment could fit in a single drawer. Constellation needs a suitably scaled solution to diagnostic and test equipment. An attempt to provide an all-in-one solution that can address the wide array of all electronics boards and their diagnostic needs would most likely result in a prohibitively heavy and difficult system. The approach proposed herein concentrates on those capabilities that would best address the greatest number of common diagnosis and repair situations, without attempting to provide a capability that will work in all situations. Understanding the types of signals indicates the type and quantity of equipment needed. For diagnostics and functional testing, channel count, frequency bandwidth, and dynamic range are important parameters. Figure 4 and Figure 5 indicate the electrical signal needs based on the typical elements of the ISS.

Figure 4 shows the bandwidth, channel count, and dynamic range for several ISS ORUs. Although the MDM (Figure 4) is distinct in that it has a large dynamic range and high channel count (480 I/O signals), it is not necessary to perform simultaneous testing of all 480 channels. By testing SRU-level circuit cards, the highest channel count can be reduced to 128 channels. By further limiting tests to a particular channel type, the channel count can be reduced to 32. This illustrates that diagnostics below the ORU level can be much simpler and help minimize the demand for large test sets. Further, SRU level diagnostics isolates the faulty component.

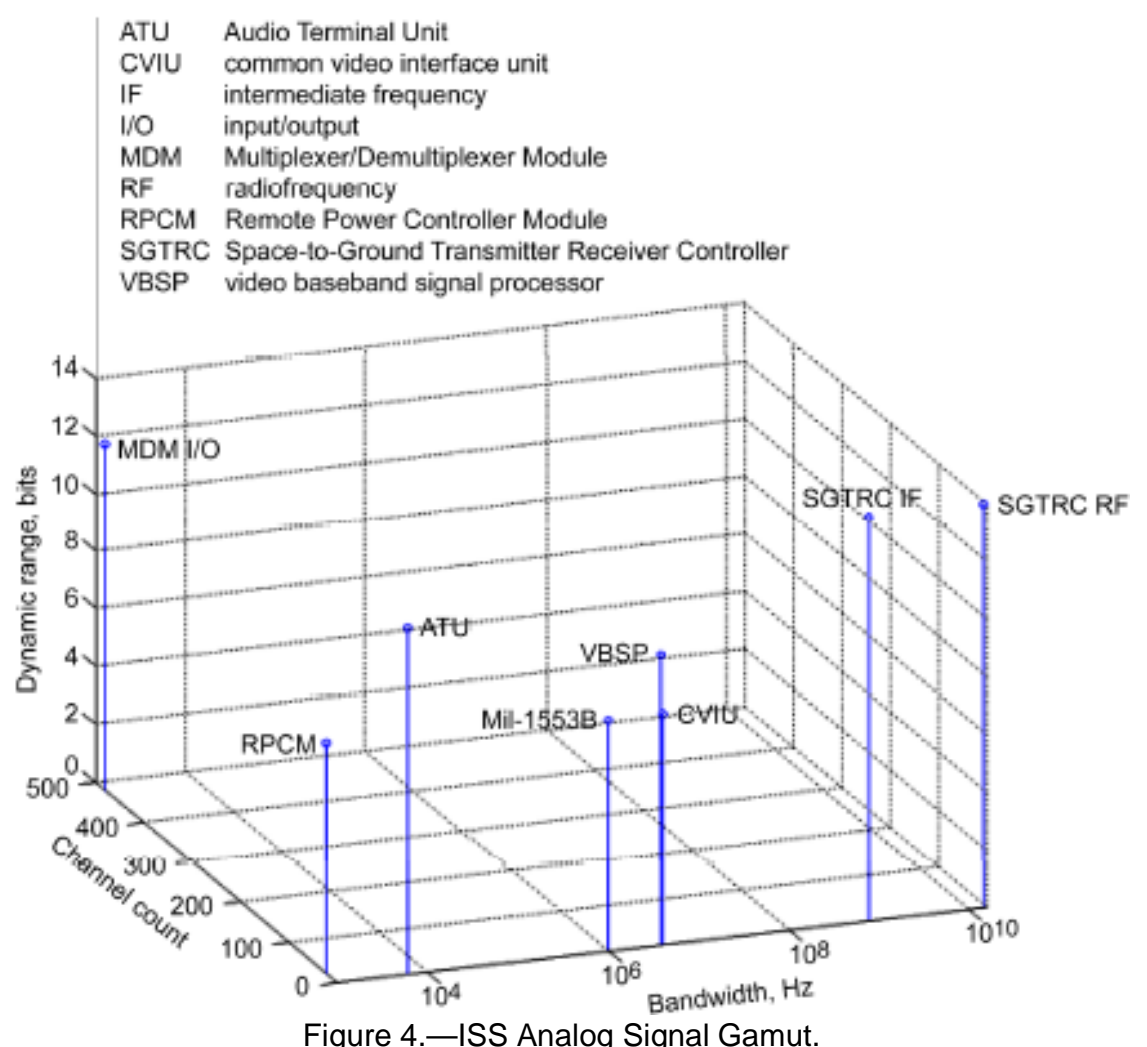


As expected, RF communication is most demanding, in terms of bandwidth and dynamic range. A down converter can be installed between the RF electronics and the diagnostics and test equipment that operates at an analog baseband frequency. By the nature of analog device technology, it is difficult to build-in self test capability. Thus diagnostics and test of analog electronics will require external equipment.

Figure 5 characterizes the digital signals of ISS electronics based on two variables: signal speed and channel count. Digital devices and their signal speeds are very subject to change with advances in digital technology. For example, the MDM bus is based on early 1990s technology and were already obsolete by the time they were deployed. The current generation digital processors have one to two orders of magnitude increase in performance. The newest generation of $\mathrm{CxP}$ avionics will exploit a high speed serial data bus such as 1394B and GigaBit Ethernet will also impact diagnostic instrumentation. Although the new architectures have very high data throughput, they also require a much smaller set of signal channels which simplifies the physical connection needs.

An important benefit of advances in processor speed and memory capacity is the greater capacity for built in diagnostics and test capability. By increasing the capability of embedded or built in diagnostics, the need for external equipment is reduced. Embedding these capabilities has little or no impact on weight and volume. Since most hardware is controlled by embedded controllers built in diagnostics should be widely used.

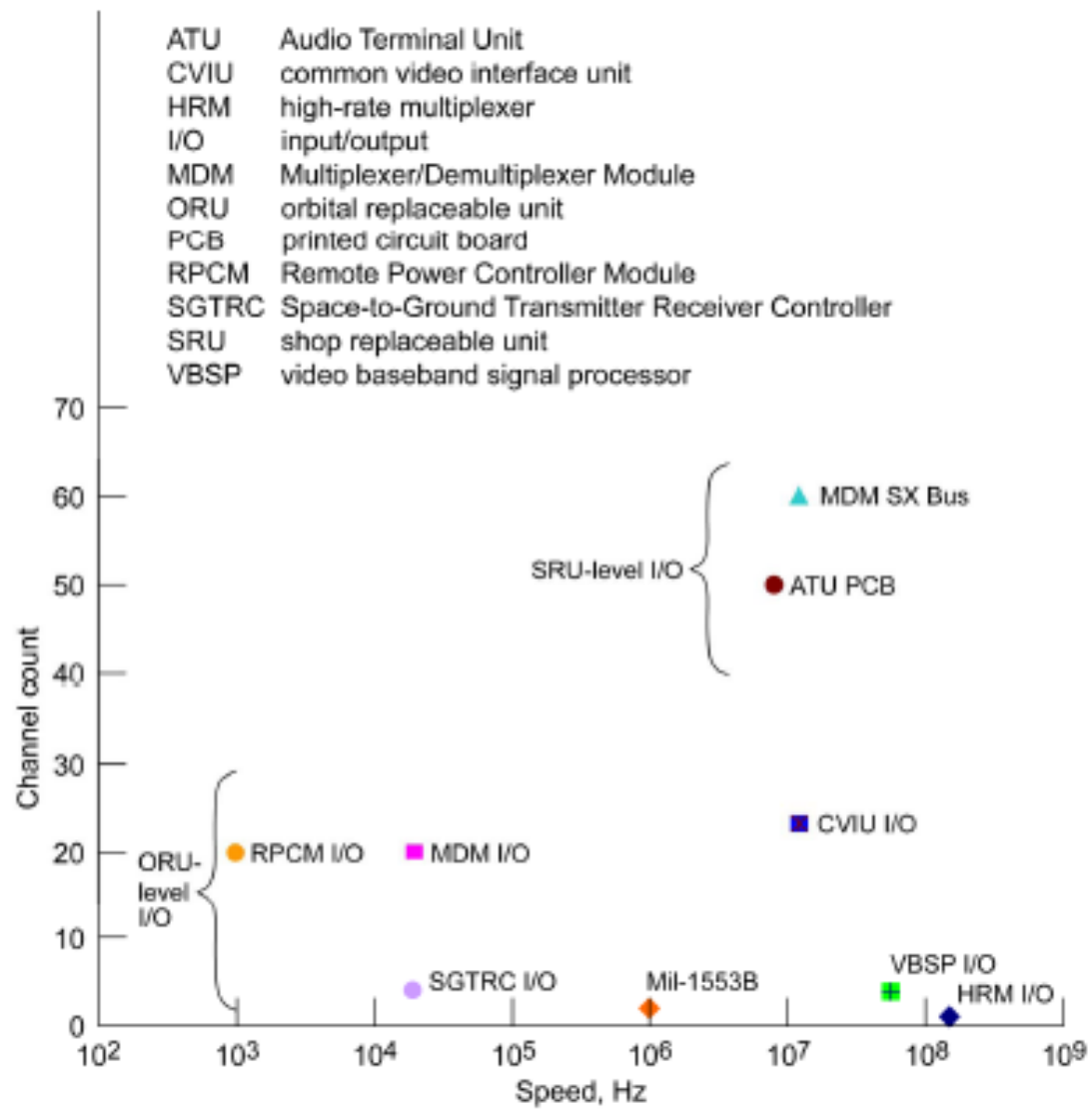

Figure 5.-ISS Digital Signal Gamut. 


\subsubsection{Manual Repair Processes}

The ease or difficulty of component-level repair depends on multiple factors, including the placement of the hardware, the ease of access, the equipment provided, the time and effort required, the basic skill set provided by the crew, and the working environment. Aside from diagnostics and test, the repair process includes retrieval, work area preparation, the mechanical disassembly of the housing, and extraction plus replacement of the faulty circuit, followed by the reassembly and reintegration of the ORU. At higher ORU and SRU levels, assembly accessibility is a dominant issue. Once a target Circuit Card Assemble (CCA) has been removed and can be freely handled by the crew, the repair becomes more dependent on the initial manufacturing materials and processes and how well the crew is equipped to rework them. In some instances, a hand-soldered assembly should readily be reworked by hand soldering techniques; however, circuits involving high density integrated circuits constructed by a solder reflow process will require more sophisticated techniques.

For manual soldering, an innate dexterity is important, but a sense of timing and patience is also required. Gauging the effectiveness of the iron's temperature setting and recognizing indications that the process is not performing properly is a skill set that only comes with practice and experience. Training can expose the crew to the fundamentals, but consistent quality results from experience and practice.

Without the benefit of constant practice, it is unreasonable to expect a crew member to achieve highquality repairs without substantial assistance. When compared to soldering processes used in manufacturing that employs programmed machinery, highly tuned processes, and constant process monitoring, the act of manual soldering is actually more complex and inconsistent. Manual soldering must be limited to applications that are simple and can tolerate some inconsistency.

Manual soldering is effective for simple tasks like soldering a discrete leaded device or attaching a wire. Both the limitations of conductive solder techniques and human dexterity contribute to difficulty as the thermal conductivity increases and the lead size decreases. For manual conductive solder repair, the ability to bring the temperature of the soldered joint up to the melting point quickly with minimal impact to the board substrate or device package is critical. Parameters that affect the heat transfer contribute directly to the effectiveness of the reliable joints. As the lead pitch becomes finer, it becomes progressively harder to solder with a conventional iron. Solder iron tips are sized to match the lead size. Smaller tips may not be able to keep up with the heat conducted out by a highly conductive substrate. Preheating is one technique that extends the operating range of the solder iron however, it also adds steps and equipment to the process.

Certain repairs are more amenable to manual repair processes without additional fixtures and are more effective than an automated process. For example, point-to-point wiring can be difficult to automate without extensive fixtures. For plated through-hole (PTH) components, the primary advantage is that devices can be positioned and, if needed, crimped so that they essentially self-fixture. Discrete and lowpin-count PTH integrated circuits (ICs) are generally easier than surface mount technology (SMT) for manual methods. Surface-mount devices can be simple to solder if the part is prefixtured by an adhesive although this becomes a deterrent later if the component is to be removed again. For ICs with high pin counts, a self-crimped PTH is much easier to handle manually than free SMT ICs. For soldered high density grid array devices like ball grid arrays (BGA) and land grid array (LGA), manual iron methods are simply not viable and such devices are exclusively reflow repair operations.

\subsubsection{Automated Reflow Processes}

Solder reflow has been universally adopted by industry because it can be easily setup and run with modest equipment and relatively few steps. Given appropriate preparations, a quality product can be produced with equipment that is essentially a sophisticated convection oven. Solder pastes are formulated to consistently activate the flux, melt, and wet out components at a predefined temperature. Solder pastes are dispensed by automated syringe dispensers or by a silk screening method that applies solder over the entire board in one operation. For reflow, CCA package factors that affect process complexity are different from those that affect the manual methods. For example, reflow is insensitive to the number of 
leads since all leads are processed simultaneously. Finer lead pitch increases the need for precise placement of the device, and metering of paste dispensing becomes more precise.

The primary difference between the reflow process used in manufacturing and the reflow process in repair is the need to localize the reflow heating to the target component without disturbing the solder of adjoining parts, which is accomplished by preheating the overall circuit board. By preheating the board to roughly $100{ }^{\circ} \mathrm{C}$ below the melting temperature, the stress on the board is reduced, the heat flux conducted away from the target component is reduced, and the time to bring the target component to reflow temperature is reduced.

Spacecraft electronic CCAs are often equipped with integrated conductive heat-sink plates, thus a preheat process is essential to minimizing the plate's cooling effect. The large conductive paths may also provide a means of preheating a circuit. It is possible to construct a heater that mates to the circuit card in the same manner as the chassis mates to the circuit card. In this case, the interface acts as a circuit card preheater.

As discussed earlier, CxP should expect future circuits to include grid array packages. Both LGA and BGA exploit the relatively large surface on the underside of an IC package. The larger real estate allows the design to provide greater lead spacing. Both LGA and BGA packages can tolerate more misalignment and void fraction that an equivalent perimeter lead flat pack. LGA can tolerate a full 50 percent misalignment and greater than a 25 percent void fraction. All these factors indicate that, for an equivalent lead count, grid arrays are easier to rework with an appropriate reflow system than conventional surfacemount devices.

In summary, component-level repair will involve integrated circuit packaging that is expected to migrate from perimeter-mounted through-hole and surface-mount technologies to ball grid and land grid array technologies. This trend limits manual solder repair methods to conductors, connectors, and simple discrete devices. High-density ball grid and land grid array devices must be repaired using automated techniques. Examination of circuit assemblies for spacecraft indicates that conductive cooling will be used throughout and circuit repair will require preheating.

\subsection{Operational Concept and Architecture for In-Situ Electronics Repair}

\subsection{Merging of Flight and Depot Operations}

For CLEAR the operational concept involves substantial human activity on Earth and in space with the intent of correcting in flight electronics problems. It encompasses existing organizations and practices while incorporating new capabilities aimed at empowering the crew in the repair of in-space electronics. The operational concept also includes scenarios where stepping through a repair process indentifies capabilities needed. The operational concept is ultimately used to define the CLEAR architecture. As described previously, the repair process is composed of three primary activities: diagnostic, repair, and functional test. Although these activities may employ the same resources and equipment, they have distinctly different objectives and, in turn, drive different requirements.

The proposed operational concept for repair combines crew-interaction with tools that will allow both manual and semi-automated operations. Generally, it is assumed that the ORU assembly will be broken down manually by the crew. This includes manual removal of ORU subassemblies, typically CCAs for electronic assemblies, and breaking the mechanical and electrical interconnections. The faulty hardware or CCA is brought to a workstation where it is to be diagnosed and/or repaired. To assure high utility this workstation could have elements which are removable and portable which may be particularly useful when moving an ORU to the workstation is impractical (for example, performing in-situ diagnostics for system troubleshooting prior to ORU removal). This workstation is only part of an overall CLEAR System Architecture.

The CLEAR System Architecture is intended to support diagnostics, repair (component replacement), and functional testing for electronic systems. For semi-automated operations, the goal is to reduce crew time usage to the extent possible. Semi-automated operations are conducted by remote control of 
equipment or machinery via telecommunications links and often referred to as tele-operations. The bulk of the human activity will be performed by ground-based engineering crews working largely offline. Depending on the application, the equipment may or may not require crew intervention during operations but the intent is to offload as much crew workload as possible and to offset the added operations involved with component level repair. The supporting crew may be formed from hardware vendors, system managers, integrators, and engineering and technical staff of NASA repair depots.

Repair operations are expected to be infrequent; the cost of keeping a "standing army" of teleoperators on standby would defeat the benefits of tele-operations. The CLEAR Operational Concept proposes that support teams be built on an as-needed basis similar to current ISS operations. Such a team, referred to here as the CLEAR Tele-operations Engineering Support Team or CLEAR TEST would provide the following:

- Specific ORU hardware and software knowledge

- Diagnostic and test expertise

- Materials and process expertise

- $\quad$ CLEAR hardware programming, verification, and validation support

- VMDB and CLEAR process library configuration management

- Hardware and spares tracking

- Coordination of flight crew operations

- Coordination of tele-operation and execution of CLEAR diagnostic, repair, and functional test operations

Many of the roles for CLEAR TEST can be provided by existing staff performing flight operations, payload operations, and logistics. Such roles are performed today for the ISS by the Logistics and Maintenance office, the ISS Mechanisms and Maintenance group (also called Operational Support Officers or OSO), and the various repair depots that service hardware for the ISS. The NASA Shuttle Logistics Depot (NSLD) and NASA Spacecraft Services Depot (NSSD) indicated that the majority of ORU repair activity is performed on units that were found to be faulty during ground processing prior to flight. The depots reported that only a fraction of the ORUs repaired are returned flight units that failed on orbit. Thus, the NASA depots will have the most recent hands-on experience and are an important asset for in-space repairs. In most cases, the original vendor will provide the most in depth understanding of the electronics ORU at the component level and, thus, will be needed to support the development of component-level repair processes.

\subsection{CLEAR Operational Concept and Architecture}

This section presents a proposed CLEAR Operational Concept and System Architecture. This architecture can be applied to electronic repair needs of missions in Earth orbit (via the ISS), the Moon, and Mars. The CLEAR Operational Concept responds to the repair needs documented in Sections 3.2 and 3.3. The CLEAR System Architecture is composed of an Earth and a Space segment (Figure 6). The Earth segment is referred to as CLEAR Tele-Operations Engineering Support Team (CLEAR TEST) and primarily involves the remote support and control of the space segment via telecomunications. The ground-based CLEAR TEST center will be similar in concept to the various science payload operations centers available today. The space segment involves a combination of manual and semi-automated diagnostics, repair, and test capabilities. The space segment hardware is collectively referred to as the Semi-Automated Repair and Diagnostic Apparatus (SARADA). 


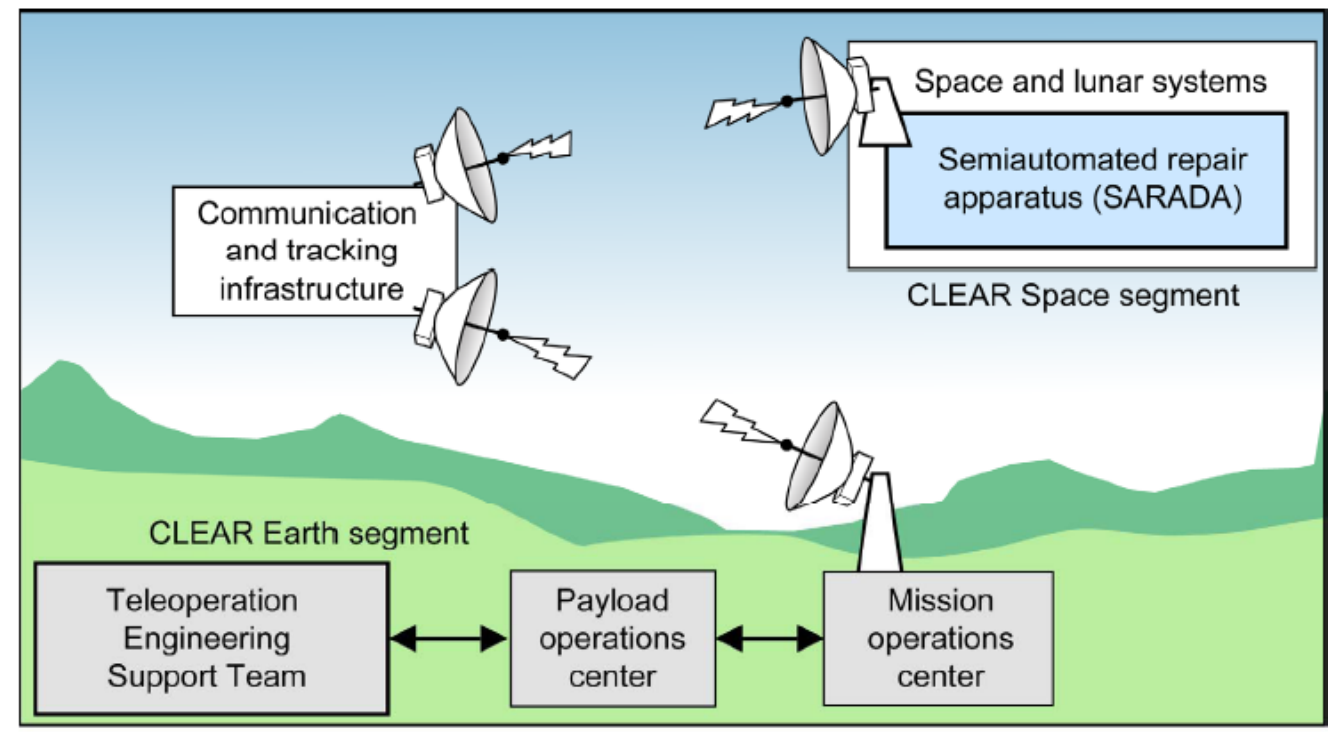

Figure 6.-CLEAR is composed of an Earth segment and Space segment that is linked via Constellation Command, Control, Communications and Information (C3I) infrastructure.

CLEAR System Architecture provides four capabilities:

(1) Tele-operations

(2) Control and Data

(3) Diagnostics and Test

(4) Semi Automated Repair and Diagnostic Apparatus.

The CLEAR System Architecture capabilities span the Earth and Space segments via the Constellation Command, Control, Communications, and Information or (Constellation C3I) infrastructure.

\subsection{CLEAR Earth Segment: CLEAR Tele-Operations Engineering Support Team}

\subsubsection{CLEAR TEST: Diagnostic, Repair, and Test Process Application Development}

Effective response to problems requires access to detailed design information and an understanding of the system history and its current configuration. The response also requires the ability to access, understand, and use the technical repair tools. The CLEAR TEST will need an array of information and an array of technical options to formulate corrective action. The development of a diagnostic, repair, and test process will involve not only the design details but the emphasis on tele-operations and remote control will require additional data and special tools, particularly if robotic equipment is used. The following sections briefly describe the database, library, and development tools depicted in Figure 7.

\subsubsection{Vehicle Master Database (Spacecraft Design Data)}

For shuttle and ISS, design details are captured in a design database called the VMDB. Diagnostic, repair, and functional tests will require detailed design and configuration information. To facilitate diagnostics, repair, and functional tests, the VMDB can be expanded to include as-built and as-repaired configurations. 


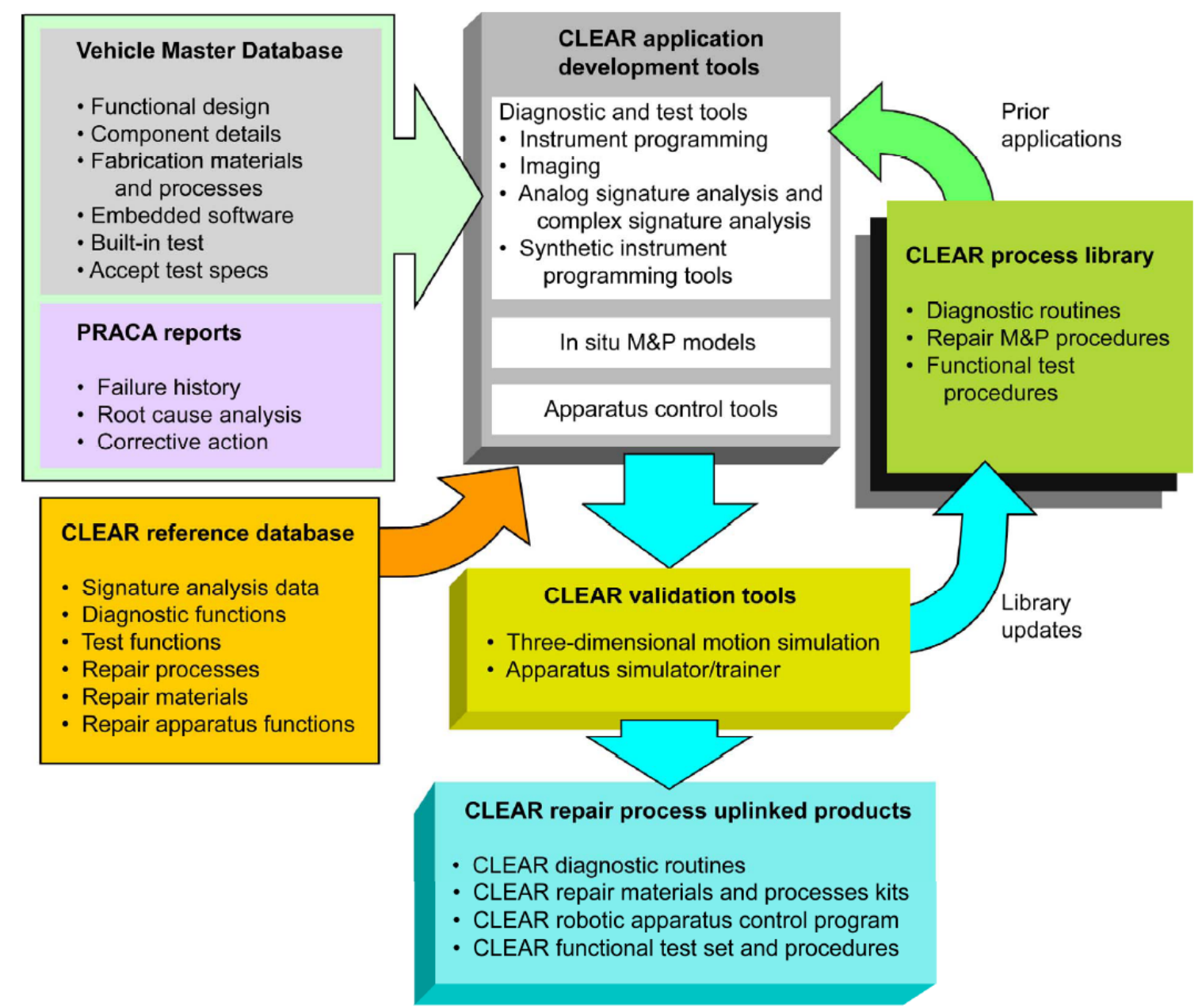

Figure 7.-CLEAR Diagnostic, Repair, and Test process development. PRACA, Problem Reporting and Corrective Action.

\subsubsection{PRACA Database (System Problem History)}

The PRACA (Problem Report and Corrective Action) system provides information regarding ongoing open-problem reports and historical closed-problem reports. PRACA reports often refer to failure analysis reports that may involve a very detailed investigation and examination of a problem's root cause. The PRACA system is very important not only as a problem archive but as a problem-reporting and tracking tool. The first time a problem is detected, a problem report is activated. From a CxP perspective, a problem report initiation will be the first indication that a CLEAR diagnostic repair and test process is needed. The problem report closeout will be the final indication that the CLEAR process is complete.

\subsubsection{CLEAR Reference Database}

A complete design database is necessary, but not sufficient, to serve all the information needs for the CLEAR task. A distinctly new data library is also required. Design databases may provide extensive information on acceptance test parameters and functional tests, but they do not generally contain information on specific repairs or diagnostic procedures and techniques. Diagnostic and repair procedures are usually generated on an as-needed basis. The CLEAR reference database will contain information unique to CLEAR System Architecture operations and capabilities. For example, circuit cards characterization information for "known-good" boards. The CLEAR reference database is composed of information not found in the design database or spacecraft problem reports. 


\subsubsection{CLEAR Application Development Tools}

The CLEAR System Architecture is expected to have significant tele-operations and will therefore be software intensive for both diagnostic and repair operations. Each repair will have unique hardware and software setup needs. Planning and subsequent programming of robotic and instrument equipment must not consume limited crew time or tie up communications bandwidth. Ground-based engineers will conduct programming to set up equipment to minimize crew time. Therefore, CLEAR application development tools also will be made available to engineers to develop diagnostic, repair, and test programs offline. The resulting diagnostic and repair programs will become part of the CLEAR process library which is discussed later.

\subsubsection{Tele-Robotic Application Tools}

These tools are aimed at programming the Space segment to perform various diagnostic and test functions for specific circuit applications. Since diagnostics and functional testing involve many of the same instruments, they will require the same development tools. In some cases, the programming involves purely electronic functions while others, such as automated probing, involve robotic motion control. The motion control parameters will be based on circuit computer-aided design (CAD) data for component location and identification and to locate registration points. Imaging development tools will allow users to develop routines to check CCA registration alignment and control the remote visual examination process.

\subsubsection{Diagnostic and Test Application Tools}

Diagnostic and test functions will rely on a new generation of "panel-less instruments" minimizes space and weight by eliminating displays and adjustment knobs and by employing a common laptop computer as the user interface. Setup of instrument parameters and coordination between stimulus signals and signal measurement must be programmed into the hardware. Analog Signature Analysis (ASA) and Complex Signature Analysis (CSA) Programming both rely on data from "known good boards." Similar to the U.S. Navy "gold disk" program, these "gold signatures" will be needed to support the diagnostics (Figure 8). If not available, a library of gold signatures will be acquired through actual tests of good circuits performed at a ground-based depot. These will be added to the CLEAR reference library for future reference.

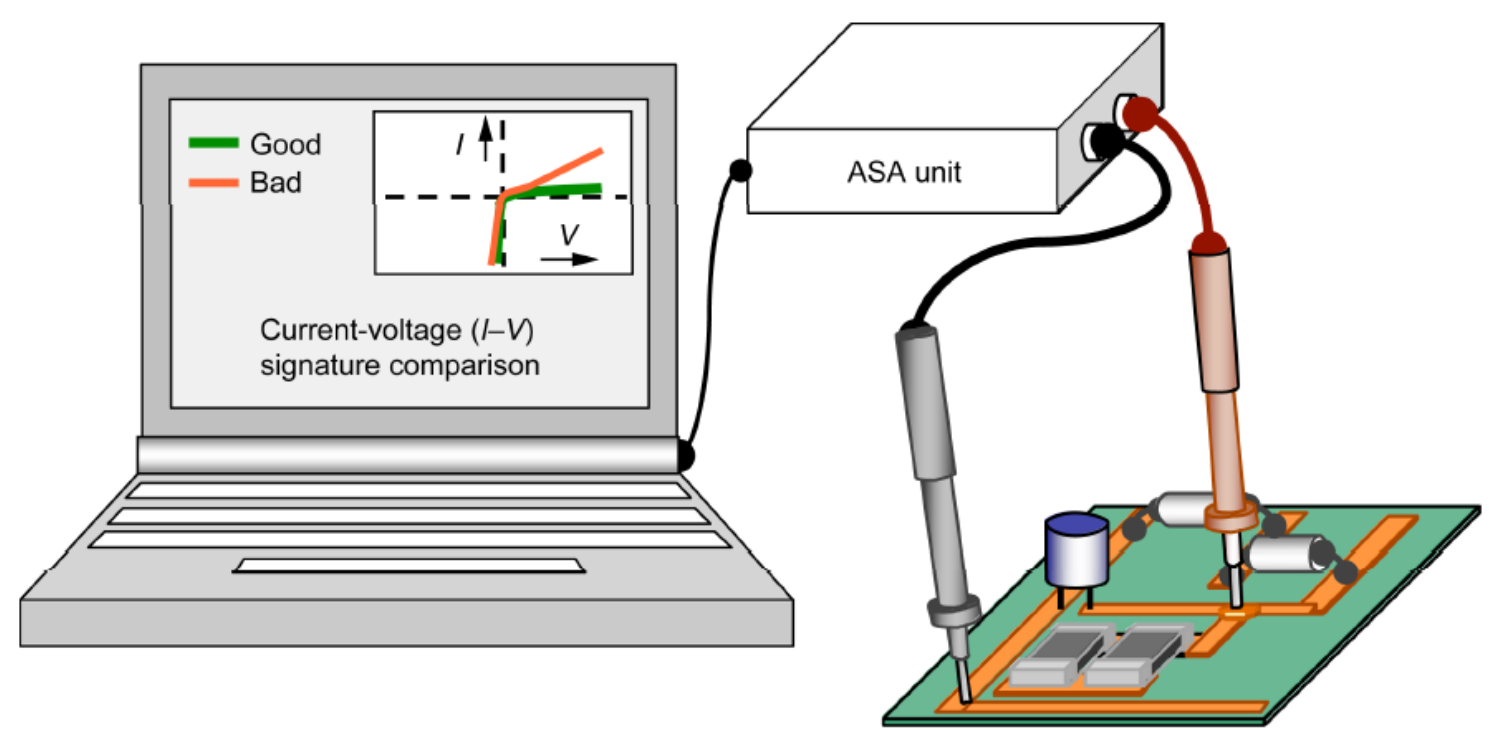

Figure 8.-Analog Signature Analysis (ASA) requires extensive characterization of "known good" or "Gold" circuit data. Such data is must be archived and managed but the result is an easy to use diagnostic tool that allows even an unskilled user to diagnose a circuit in space. 


\subsubsection{Synthetic Instrument (SI) Application Tools}

Synthetic Instruments technology provides a versatile but compact instrument capability. This is described further in the diagnostics and test architecture. In many ways, synthetic instrumentation is based on the premise that many instrument functions are digital or can be performed by digital circuits, which implies that instrument functions can be operated as software. To achieve instrument-level performance, it is necessary to embed software into field-programmable gate array (FPGA) that allows designers to exploit data pipelines, parallel processes, and other signal processing techniques that give conventional instruments their high performance. A set of tools will be needed to create an instrument model and then perform the hardware programming. Ground-based simulators of the flight hardware will be needed to develop and validate the code.

\subsubsection{Low-Gravity Reflow Application Tools}

Solder Reflow is used for removing components and installing new components. The process typically employs a solder paste with heat-activated flux. A predefined temperature profile is required to ensure proper flux activation and solder reflow, yet it minimizes the excess temperatures that may damage the circuit. Solder repair processes may have a dependence on gravity where voids in metal solder joints are more prevalent than 1-g and require certain adjustment to the process. Gravity also eliminates normal buoyancy driven convection which alters the thermal behavior of the process. To account for gravity effects the reflow thermal profiles developed in normal gravity will need to be adjusted for reduced gravity. Therefore, reflow modeling tools will include low-gravity effects to adjust heating profiles to assure a reliable repair and prevent circuit damage.

\subsubsection{CLEAR Integrated Validation Tools}

Before complex commands are uplinked to the Space segment, the code must be validated. The complex interactions among the instruments, robotic hardware, flight crew, and repair targets involve so many details that it is easy to overlook a detail that results in damage to hardware or injury to a crew member. Industry uses development tools that can also perform simulation and serve as an initial validation of the process. However, the interaction between robotics, instruments, and crew members will require a simulation of the overall process. If substantial crew interaction is required, then a live simulation using a high-fidelity mockup of the system will be needed. The simulator and/or trainer would support this simulation and also serve as a trainer for flight crews.

\subsubsection{CLEAR Repair Process and Uplink Products}

The final products of the CLEAR development and validation effort are primarily software to configure and control hardware as well as acquire data. For specific ORU and subassemblies, palletized repair component kits could be preassembled and stored in stowage. Most repairs will consume only a few pieces of any repair kit and could be replenished on an as-needed basis.

\subsubsection{CLEAR Process Library}

The CLEAR process library represents the accumulated knowledge for the diagnostics, repair, and test of circuits. Unlike other databases, this library is intended to contain reusable control codes and setup parameters. Much like an industrial CNC library, it will contain the programming code and parameters for specific component-diagnostic and repair operations. Because diagnostic processes search for an undetermined cause, a variety of diagnostic routines may be used to determine a specific fault. Over the life of the circuit design, multiple independent faults may occur. Therefore, we expect to accumulate a large library of diagnostic routines. Likewise, a procedure may be needed for every repair of a given component type. Therefore, like diagnostics, a significant library of repair procedures can be accumulated. Finally, a delta (subset) of acceptance test procedures will be used to verify that the circuit has been successfully repaired. It would be useful to have a template process that covers setup operations and contains commonly used processes. Should a similar fault reappear, a great deal of effort will already 
have been captured. Over time, the number of team members required can shrink as the library grows and experience makes the team more effective.

\subsection{CLEAR Space Segment}

\subsubsection{CLEAR Tele-Operations}

Regardless of the repair method (manual or automated), it is essential to define space and groundbased activities related to the repair process and the telecommunications infrastructure that links them. Repair is an off-nominal or contingency operation and there may be considerable risks to the crew and mission. The wide diversity of systems makes it impractical to have each crew member familiar with the level of detail required to diagnose and repair flight hardware. The crew must be backed by ground-based expertise and a knowledge base capable of resolving the problem, creating and coordinating crew procedures, and programming equipment to execute corrective actions. Historically, NASA has augmented the flight crew by employing extensive telemetry, communications, and monitoring of systems by mission operations centers. Science payloads have been supported by remote payload operations centers that provide access to science instruments and experiment payloads, without adding a burden on the primary flight system operations.

The CLEAR Tele-Operations concept is also intended to minimize dependency on real-time interaction. Real-time interaction would be feasible for some operations. However, the process of diagnosing faults, testing, and setting up a repair is prone to long pauses while support staff assess data and consider the next step. Considering the high value of both crew time and communications, it is more practical to perform as much of the process offline as possible. Similarly, microgravity experiments aboard the space shuttle and ISS were extensively preplanned and automated with optional breakpoints in the experiment procedures to accommodate changes based on near real-time assessment of experiment data. The CLEAR Tele-Operations is envisioned to operate in the same manner as a Tele-Science Center to minimize operational costs.

CLEAR Tele-Operations will operate the flight hardware in manner frequently used in flexible automated equipment commonly used in short-run manufacturing operations. To compensate for the high cost of labor, manufacturers leverage machinist skills by capturing machine operating parameters as special CNC (computer numerical control) code files. The CNC machinist no longer manually operates the machine tools but instead creates a script-like program of simple and compact machine "G-codes" that instruct the machine to load specific tools, position the work piece and execute machining operations in manner similar to what a manual machinist would perform. Although the machining process is automated, part mounting, tool loading, machine alignment, and consumable resources are all handled by a manual set up. On occasion the automated operation has preprogrammed pauses to allow the machinist to check progress and inspect the hardware. Thus CLEAR operates in a similar "semi-automated' manner. The primary difference is the programming will be performed on the ground by the CLEAR Teleoperations Engineering Support Team and not the flight crew.

The mechanical portion of the CLEAR SARADA, shown in Figure 9 configured in a repair mode, will be designed to accommodate both manual and automated operations and provide repair, along with diagnostic and test probing capabilities. For the most part, CLEAR SARADA will not operate under remote real-time control. Programming and subsequent verification will be performed offline and will avoid unnecessary consumption of crew and tele-operations time. To provide efficient preprogrammed control and to accommodate flexibility, CLEAR TEST engineers will develop and verify prescripted diagnostic and repair routines that may include decision branches at key points.

\subsubsection{CLEAR Control and Data}

The CLEAR Control and Data (CC\&D) system architecture serves as the data backbone of the Space segment. The CC\&D system must provide for incremental development of the repair-related technologies. It must be established early and yet resist obsolescence. A local-area network (LAN)-based architecture is 


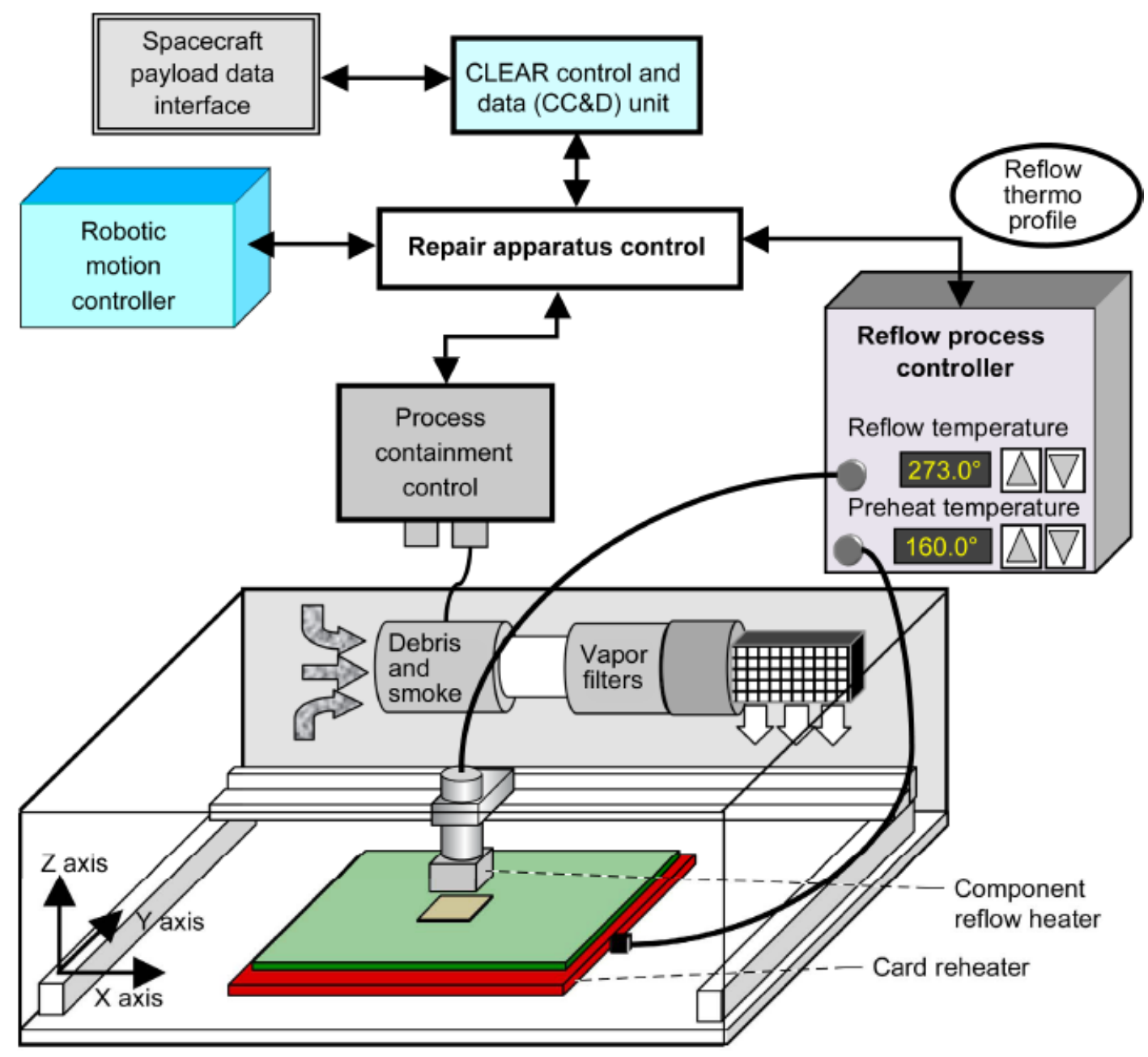

Figure 9.- The mechanical portion of the semi-automated Repair and Diagnostics Apparatus (SARADA) also requires process containment to protect the crew cabin environment.

inherently an open architecture, less restrictive than proprietary architectures, and considered the best option for long-term stability. The LAN-based data backbone is consistent with a plug-and-play approach that provides ease of integration and incremental growth.

Figure 10 shows many major SARADA functions and illustrates the interconnections where commands, data, and power flow. The CC\&D controller is both the main controller and master of the data network or data backbone of the system. This backbone function is essential to the incremental development strategy and must be defined explicitly prior to incremental hardware development. The CC\&D controller is also the gateway for tele-operations. Each block in Figure 10 has a smart subsystem controller that also provides network interfaces to the block above and below it in the system hierarchy. Ideally, the blocks would provide a plug-and-play capability that would allow additional blocks to be added without requiring a massive reconfiguration of the system. The best way to accommodate plugand-play is with LAN protocols.

The CC\&D controller is linked with the spacecraft's Command and Data Handling (C\&DH) network. The channel on the spacecraft side would be the branch of the C\&DH system that supports payloads. Using payload channels, instead of primary system channels, eliminates the concern that CLEAR transactions would consume bandwidth needed for mission support. Since the payload command and data channels pose no risk of system hardware interference, the CLEAR TEST center can operate independently. 


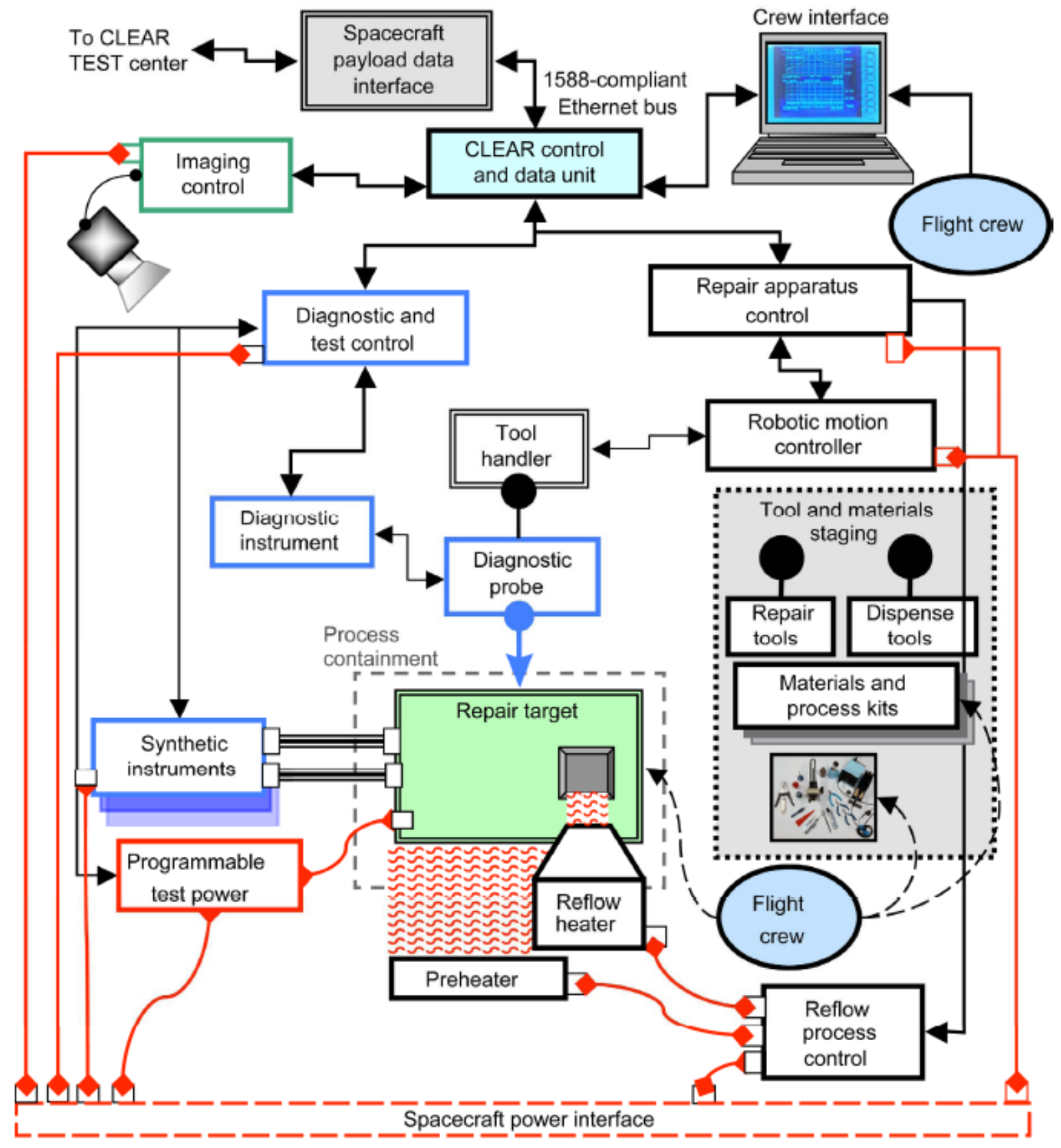

Figure 10.-Functions of the Semi-Automated Repair and Diagnostic Apparatus (SARADA).

The crew will interface the system through an available laptop computer. The crew interface may be used for monitoring the CLEAR process, archiving instrument data, and performing data processing required to analyze and display data. The crew interface will provide the graphics display for CLEAR process setup and operating procedures. The crew interface will provide a CLEAR process library that will include background information and refresher training for the crew. The crew interface also will provide the crew with an override authority to interrupt or halt CLEAR processes and will provide an emergency stop function for the crew.

Other key components of the CC\&D are the controllers for imaging, diagnostic and test functions, and the repair apparatus. These functions are described more in the sections below. 


\subsubsection{CLEAR Diagnostics and Test}

The CLEAR Diagnostics and Test (CD\&T) system architecture provides instrumentation for diagnostics and test. Diagnostics is used to determine and isolate a system malfunction to the component level and determine the root cause. Testing (functional) is aimed at verifying that the repair has restored full function and verifying that the repaired assembly is suitable to return to service. Although diagnostics and test are different activities, they often use similar functions and the same instruments. Thus, diagnostic functions and test functions are considered as simply different applications of a single CD\&T system.

Due to payload constraints the allocation of size, weight, and power for diagnostics is expected to be much smaller than typical automated test equipment that may occupy multiple racks. Instead of racks, an accommodation equivalent to single drawer $\left(0.2\right.$ to $\left.0.3 \mathrm{~m}^{3}\right)$ is more viable. This rules out building as systems form conventional bench top instruments. CLEAR Diagnostics and Test architecture considers strategies that will pack as much capability into drawer space as possible. Synthetic Instruments is an approach that the Department of Defense (DoD) has invested in. Described in more detail in technology development section, the approach seeks to minimize equipment by employing technologies that allow the user to "synthesize" instruments on demand. Synthetic Instruments is expected to be software intensive and this means that SI applications can be developed and stored in memory and called up when needed. This also means specific circuits diagnostics and tests routines will be uplinked code rather than launched equipment.

For initial fault isolation that also minimizes crew demands CD\&T will exploit Diagnostic Signature Analysis techniques. DSA uses an intuitive graphical comparison technique that expands on the U.S. Navy's success in with Analog Signal Analysis. Described in the technology development section, this approach should allow crew members with relatively low electronic diagnostic skill to successfully diagnose and isolate electronic faults.

Functional tests requires a wide array of measurements, external power, and emulation of external system interfaces. To the extent possible SI will also provide emulation support for low power external signal emulation. Equipment like load simulators are a serious threat to CD\&T size and mass. To support tests requiring massive loads CLEAR is considering methods that will allow electronics to be tested by tying into parent system. Special smart interface devices may be used to act as in circuit interrupts or isolators in case a faults that threaten the system

Imaging is essential for examination, process control, and post-repair evaluation. Imaging data usually demands high data rates and mass storage. A separate imaging controller provides dedicated circuits that minimize the impact on control loops and other instrumentation. Although there are a variety of imaging devices potentially available from visible light to infrared (IR) and even X-ray, this system can be used to convert images to a common digital format.

\subsubsection{CLEAR Repair Apparatus}

Component-level repair may be performed manually and will always be an option when human crews are available. Development of manual repair capabilities by the CLEAR team are further discussed in Section 4.0. However, many repair operations are best handled by dedicated preprogrammed equipment designed to reproduce many of the conditions used in manufacturing. A fully automated process such as in manufacturing requires special fixtures and equipment, along with a great deal of experimenting or system "tuning." For single unit repairs, a mix of manual and automated operations is more practical and is most likely to achieve successful repairs within the spacecraft weight and volume constraints. Certain operations, such as wiping away process residues, are simple if performed manually but can be very difficult to accomplish by robotic means. Heating devices to narrow temperature bands and then quickly and precisely placing them demand automated operations. Semi-Automated repairs reduce the demand for high skill while increasing productivity.

The CLEAR Repair Apparatus (CRA) system addresses the repair processes that are beyond the capability of manual tools and the crew skill level. The CRA borrows heavily from industry experience with automated and robotic technology. Figure 9 shows the SARADA configured in a repair mode where 
a reflow system is integrated into a Cartesian robot for positioning. Reflow heat may be delivered as hot gas or as IR radiation, or use a combination of both. The CRA architecture is intimately tied to the CTO architecture for operations.

In addition to reflow hardware which uses varied combinations of motion and heat application, other automated processes would include various other repair needs. This includes the manipulation of diagnostic probes which may be may be passive meter or scope probes or they may be actively injecting signals for DSA applications. Imaging cameras will support visual examination and process monitoring needs. The imagers will be used in setup to locate key registration points and check the alignment of the target circuit with the repair apparatus. The camera to ride along on a tool handler as seen in commercial probing stations and certain pick-and-place machines. Many of the tasks and tools used in manual repair will need to be duplicated for semi automated operations. Motorized rotary tools for drilling, cutting, grinding, and cleaning are safer and more precise if performed robotically. For example, using rotary tools to remove conformal coatings without damaging the underlying circuit requires a rigid tool handling carriage and precise depth control. Finally, automated pick-and-place is important for accurate placement of components. Component pallets present the components for easy pickup by the pick-and-place component handler.

Repairs performed by manual or automated means will benefit from proper staging of the hardware. Tools used by the tool-handling robotic mechanism need to be staged in pre-assigned positions so that a robotic mechanism (or human) can quickly locate them. Unneeded items will have to be kept clear of the work area. Since space will be limited, components, tools, and materials should be palletized so that they can be placed in the work area quickly and removed quickly.

Throughout the repair process, there are operations that create debris, vapor, and smoke. A basic glovebox containment will suffice for most applications. The majority of solid debris and vapors can be controlled by circulating the air through filters. Filters however, are consumables so processes must minimize debris and vapors. Containment of the small amount of cleaning and rinsing liquids can be controlled by using saturated wipes. The containment shown in Figure 9 also will include glove ports for manual operations. On ISS, there are existing glovebox facilities such as ISS Microgravity Science Glovebox (MSG). The CLEAR SARADA could be simplified to fit into a glovebox and minimize the overall up-mass required. It is likely that the lunar facilities also will have similar glovebox containment available.

\subsection{Progress Towards Development of Component Level In-Space Electronics Repair}

\subsection{Incremental Development}

The CLEAR project objectives imply providing a NASA depot-level capability squeezed into the constraints of a spacecraft in a remote environment with no direct precedence. There is an array of technologies and techniques to consider, and an innovative approach is needed to render them compact, lightweight, and compatible with space. Some requirements can be addressed by analysis and tradeoff studies. Innovative, but untested, techniques will require analysis, lab work, and low-gravity tests to validate them for space.

With no accurate way to predict which circuits will need repair, there is a tendency to cover every possible repair option. This would result in a project with huge development and coordination costs, as well as, a long requirements-definition phase and development contracts. Program managers will attempt to contain cost by pushing an aggressive schedule and selecting low-risk off-the-shelf technology. The resulting product could be a collection of technologies that is a poor match with the operational environment and poorly understood by its users. If users find equipment to be unwieldy, it will provide very limited utility.

Operational experience will reveal the failures and the diagnostic, repair, and test needs. Experience also will provide the opportunity for an appropriate mix of crew, robotics, and tele-operations capabilities 
to evolve. Incremental development will accommodate the development of techniques with varied levels of complexity. Therefore, it is essential to capture "low-hanging fruit" with early flight experience, such as manual soldering methods from Station Development Test Objectives (SDTOs) aboard ISS. More challenging and complex repair methods that involve automated or robotic solutions will have a longer time to evolve.

A system engineering approach that focuses on $\mathrm{CxP}$ objectives is needed to assure that the end product meets the program needs. Semi-automated repair, diagnostic operations, repair, and functional test will depend on ground support and tele-operations. These tasks will be more complex than simply building an isolated apparatus. Thus, the overall CLEAR System Architecture is intended to be developed as a series of technology packages. The last package must be compatible with the first package. The initial flight package must include tele-operations and the data backbone architecture of the system. This requires that decisions for this data backbone must be made at an early stage of development.

Because of the long development cycle and the continuous advances in data system technology, the CLEAR System Architecture must anticipate obsolescence to ensure long-term viability and minimize life-cycle cost. The CLEAR System Architecture will employ two approaches to combat obsolescence: (1) a local area network will be the data backbone of the system and (2) an evolving technology called synthetic instrument (SI) will be exploited.

As with ISS, CxP will have constraints for payload, crew time training, and communication bandwidth. Therefore, tele-operations capability will be developed to fit within existing capabilities. To prevent adding operational burden to the program, the CLEAR System Architecture will use the telecommunications channels used to support science payloads. This will prevent competition between CLEAR and on-going flight operations for high-priority command and control channels

\subsection{Reduced Gravity Aircraft Testing}

To gain confidence in the reliability of soldering as a standard repair process for space missions, it is important to assess the effect of reduced gravity on the soldering process. Prior to the CLEAR project, a group of researchers conducted a series of reduced-gravity soldering tests utilizing NASA's KC-135 research aircraft. The work, which began in 2001, involved 8 flight weeks of testing and spanned over $3 \mathrm{yr}$ (Refs. 13 to 17). The primary repair configuration investigated used a plated through-hole (PTH) configuration. Results of these tests characterized changes in the solder joints, including geometric changes and increases in internal porosity, which can increase by as much as a factor of 3 , as a result of the reduced gravity environment. The sources of the increased porosity appear to be flux vapor which was trapped in the molten solder, and entrapped moisture in the circuit board itself. Several techniques were tested to mitigate the porosity increase, including alternate techniques for flux application and demoisturization of the circuit board prior to soldering.

Continuing this previous work, the CLEAR team conducted a series of solder tests aboard NASA's new microgravity C-9 reduced gravity aircraft. This work included the examination of various solder and flux combinations, most focusing on the use of no-clean or water-clean fluxes with eutectic solders, in preparation for an ISS experiment investigating soldering (see Section 5.3). The analysis included discriminating between samples based on the quality of the reduced gravity environment as well as the quality of the solder joint based on NASA standards. Acceptable samples were nondestructively analyzed for void formation. Results from this work were presented elsewhere (Ref. 11) but were consistent with results from previous testing. These results will help develop criteria for selecting solder, flux, and techniques to be used for in-flight repair of electronics.

\subsection{Recent Spaceflight Experiments}

As discussed previously in Section 3.2, the ISS has available a basic soldering kit, tools, and workarea which can be used for some simple repairs. Augmenting these existing ISS tools with additional repair tools, the CLEAR project conducted two space-flight experiments aboard the ISS to investigate and 
demonstrate component-level repair. The first experiment, called Soldering in Reduced Gravity (SoRGE), investigated the basic processes of soldering in a microgravity environment in a through-hole configuration. This experiment built on the results of the reduced gravity aircraft testing described in Section 5.0. The second experiment, called Component Repair Exp-1 (CRE-1), demonstrated key aspects of the physical processes of end-to-end component-level repair of a circuit board. These include conformal coating removal, component removal, board clean-up, and component replacement. Details and results of from these experiments are presented elsewhere (Ref. 12).

\subsection{Recommendations to Help Enable Manual Electronics Repair}

As a result of the spaceflight experiments described in the previous section as well as study conducted with the U.S. Navy, the CLEAR team is preparing a report (Ref. 18) that provides recommendations to help enable manual replacement of electronics at the component-level. The report discusses the current ISS repair capabilities followed by recommendations for future missions. These recommendations include hardware and tool requirements, containment options, and crew training. A brief synopsis of these recommendations is presented here.

To enable component-level electronics repair in future manned space missions, crews will require significantly more capability than currently available aboard ISS. The entire repair process encompasses fault diagnostics, the physical repair, and acceptance testing post repair. Additionally, crew members need adequate skills based training to be able to respond to wide variety of potential problems. Each area (diagnostics, repair, test, and training) requires more capability and forethought to enable future crews to conduct repairs in a manner consistent with the constraint of spaceflight. One of the primary constraints is that of available crew time. Crewmembers primary mission is to conduct the science and exploration tasks assigned. Repairs, although inevitably necessary, should not require the majority of the available crew time to conduct.

Fault diagnostic and post-repair functional test concepts are as discussed previously in this document. These include a combination of increased built in test (BIT) capabilities, signature analysis devices, and synthetic instrumentation which can be programmed to meet a specific diagnostic and test need. If a semiautomated system such as the SARADA is not yet available, manual probing (as shown in Figure 9) for fault diagnostics would be required. Testing needs would likely be some subset, if any, depending on the equipment available for the functions shown back in Figure 1.

For the physical component-level repair, additional tools to those already aboard ISS are required. For most repair applications, a higher-end, electronically-adjustable, soldering stations (e.g., those that can be adjusted in $1^{\circ}$ increments to satisfy the needs of the actual application) is recommended. Furthermore, soldering irons which do not require calibration are desired. Should a higher-temperature tip be problematic to certify for use in spacecraft, another alternative is a backplane heater (although this increases the equipment to be carried). The soldering iron tip does not have to be as hot as in the case where the circuit board is at room temperature, because the heater provides most of the energy instead of the soldering iron tip. Heating the circuit board with the backplane heater also reduces thermal shock to the board by reducing the temperature gradient between the board components and soldering iron tip. The available tips in the U.S. Soldering Kit only include one style tips-a screwdriver shape. Smaller or other shapes are required to more easily work with many component types including surface mount technology (SMT). A number of tools are required to extract solder and maintain the solder extraction equipment

While the existing ISS IVA Toolkit has a number of hand tools available, most are not suited for use in electronics repairs due to size. Smaller electronic components require tools to manipulate the smaller components. These include cutters, pliers, tweezers, conformal coating removal tools, lead-forming tools, ESD protection, and wire strippers. Fiberglass sticks, dental tools, and even toothpicks are needed for removing conformal coating, reapplying conformal coating, or as a tool to position a component on the circuit board. The fiberglass in the stick will not wear away as readily, as a wooden stick would, when removing conformal coating. The stick will also limit damage due to shorts or static build-up during use. 
Other aspects of tools include designs which reduce user fatigue as well as ensuring that the tools are not mishandled or used for applications other for which they were intended.

The needed materials and supplies include solder, external solder flux or flux pen, and de-soldering wick. If alcohol is used for cleaning or other applications then containment of the alcohol vapors from the environment is required. Additional supplies which may be part of a general supply kit include tissues (lint-free), toothpicks, and extra fuses. Due to the increases in void defect formation described earlier (Ref. 13), an optimum solder chemistry may be recommended pending the results of the SoRGE experiment. Note that the 60/40 Rosin Activated (RA) type solder in the current kit is not recommended due to the highly active rosin flux core and no way to easily remove the flux residue due to restrictions on alcohol use. In general, solders and processes which minimize cleaning such as the use of no-clean flux are considered optimal.

The tool recommendations discussed thus far focus on the removal and replacement of electronic components. Other electronics repairs may arise which require specialty tools. One example is repairing a broken trace or land on a circuit board. Such repairs require the use of a rotary hand tool to excavate around the trace or access an internal trace. Tweezers and picks are required to manipulate small replacement materials or debris. Furthermore, chemicals are needed to clean or bond replacement parts, requiring tools to apply and handle the chemicals. While some tools, such as the rotary hand tool, may be available as a part of a general tool kit, most other tools for this or similar tasks will not, and must be included in a dedicated electronics repair tool kit. The amount of epoxy required to perform conductor repair, both surface and sub-surface, may be too much for the closed environment to handle. As a potential alternative, surface conductor repair can be attempted by soldering the replacement in place and using the reapplied conformal coating to secure the repair.

The existing containment enclosure, used with the MWA on the ISS, has a number of shortcomings that are specific to conducting component-level repair of electronics. The following recommendations focus on design considerations for a new containment enclosure. This discussion will also examine visual aids which may be included in a future containment enclosure, or as stand-alone instruments with applications beyond electronics repair.

Perhaps the single most important recommendation is to have an enclosure which will allow crew members to comfortably see and manipulate small parts within the enclosure. Terrestrially, technicians do not normally use an enclosure and are typically within $12 \mathrm{in.} \mathrm{of} \mathrm{the} \mathrm{circuit} \mathrm{card.} \mathrm{With} \mathrm{regard} \mathrm{to} \mathrm{the}$ physical characteristics, the containment enclosure should have rigid sides, to avoid problems with visibility through the current soft sided enclosure, and a clear window to allow easy viewing of the CCA. The enclosure should still be collapsible to aid in stowage (although a less setup time would be highly desirable as the current design requires at least $30 \mathrm{~min}$ for setup). As with the current design, the enclosure should have electrics feedthroughs and hole for a vacuum cleaner. The enclosure should be sized to accommodate most, if not all, circuit boards a crew member would work on, with enough volume to accommodate the soldering tool, board clamp, backplane heater, or other tools and instruments that may be necessary.

Visual aids can improve the visibility for the crew while performing a repair. Many of the component legs and circuit card pads manipulated in a repair task are small, and working with these small parts may be difficult or impossible, and taxing on the crew member, if using only the naked eye. Visual aids that can alleviate or eliminate these problems include magnifying lenses, which can be used in a standalone fashion, as part of the enclosure wall, incorporated in lighting, as a visor the crew member wears, or in other ways. Low power microscopes (on the order of $20 \mathrm{X}$ magnifications) are commonly used in ground laboratories and workshops, and can be used with the flat sides of an enclosure in a space vehicle or habitat setting. Video cameras with macro lenses may also be used to magnify the work area, shown in Figure 8 for example, with the benefit of recording the repair session for future analysis. Mission planners should include multiple choices among the visual aids as well, allowing crew members to be flexible in terms of using the appropriate magnifier for the task at hand, available light levels, transmitting or recording video, and personal preference. 


\subsection{Technology Development Concepts}

\subsubsection{Synthetic Instruments}

Synthetic Instruments (SI) refers to an approach that exploits software and reconfigurable hardware to maximize the flexibility of test systems. The motivation behind SI is the need to reduce the size and weight of instruments and related hardware used in the diagnosis and test of electronics. It is not uncommon for a specific ORU to require multiple racks of equipment. Department of Defense (DOD) studies consider automatic test equipment (ATE) as a primary contributor to the high life-cycle cost of avionics equipment (Ref. 19). ATE life-cycle cost can become an increasingly expensive part of avionics cost over time as hardware becomes obsolete. The studies also determined that test software is a major component of avionics and test equipment cost. Hardware and software obsolescence also tends to drive up cost, particularly for proprietary systems. These DOD studies prompted development of nontraditional concepts for ATE to keep life-cycle cost down. SI is conceived as a way to make ATE equipment more universal and easier to update remotely.

SI is implemented on generic hardware, typically Field Programmable Gate Arrays (FPGAs). FPGAs are designed to provide a vast array of logic gates that can be configured to provide distinct data and control functions. SI is different from virtual instruments, or VI, which employs purely software control of digital and analog I/O and rely on computers central processing unit for timing, control, and data handling with a significant performance penalty. Unlike software executed by microprocessors, the SI programmed into the FPGA executes at extremely high speeds and with high levels of parallel processing. The FPGA can be reconfigured, on demand, as a completely new SI. The latest generation of FPGAs can provide real-time signal processing which was once the domain of digital signal processors. The main advantage of SI is that it allows us synthesize high performance instruments on an "as needed" basis.

The strategy behind SI is to allocate any digitized functions to software. Innately analog functions, such as sensors, analog signal devices, and power sources are handled by modules that can be reconfigured electronically to provide wide operating ranges. In addition, to handle the vast variety of connector configurations, SI provides an upfront analog switching matrix that routes signals from a target test circuit to a selected analog instrument module. The CLEAR team considers SI (Figure 11) as part of a strategy to provide both diagnostic and functional test capability for a wide range of spacecraft electronics using only a small amount of physical hardware.

\subsubsection{Diagnostic Signature Analysis}

Diagnostic Signature Analysis (DSA) is a general term coined to indicate diagnostics by comparison of characteristic signatures. Analog Signal Analysis (ASA) refers to a fault finder technique widely used by the U.S. Navy for ship board diagnostics. By graphically comparing a test signatures from a faulty circuit with a known good circuit a relatively inexperienced technician can quickly identify circuit faults. This capability is desirable for flight crews where crew training is focused on mission objectives rather than system troubleshooting. ASA however, has limitations, particularly for high frequency circuits. Therefore, DSA will likely incorporate a new approach called Complex Signature Analysis (CSA).

CSA is an extends the concept of ASA by exploiting the capability of a vector network analyzer. Not to be confused with network protocol analyzers, a vector network analyzer tests networks of components in high-frequency signal circuits. Vector network analyzers share certain capabilities with a spectrum analyzer. These devices are generally used for high-frequency or RF circuits, where operating frequencies can range from $10 \mathrm{kHz}$ to $110 \mathrm{GHz}$.

A vector network analyzer measures the frequency domain properties of component networks rather than single components. It injects a signal into the network and measures the signal reflecting back from the circuit and the signal transmitted through the circuit. These analyzers are commonly used to test highfrequency amplifiers, filters, and tuning networks and can also test piezoelectric devices like crystal resonators. They operate at very low wattage and inject signals into passive networks or active devices like amplifiers. 


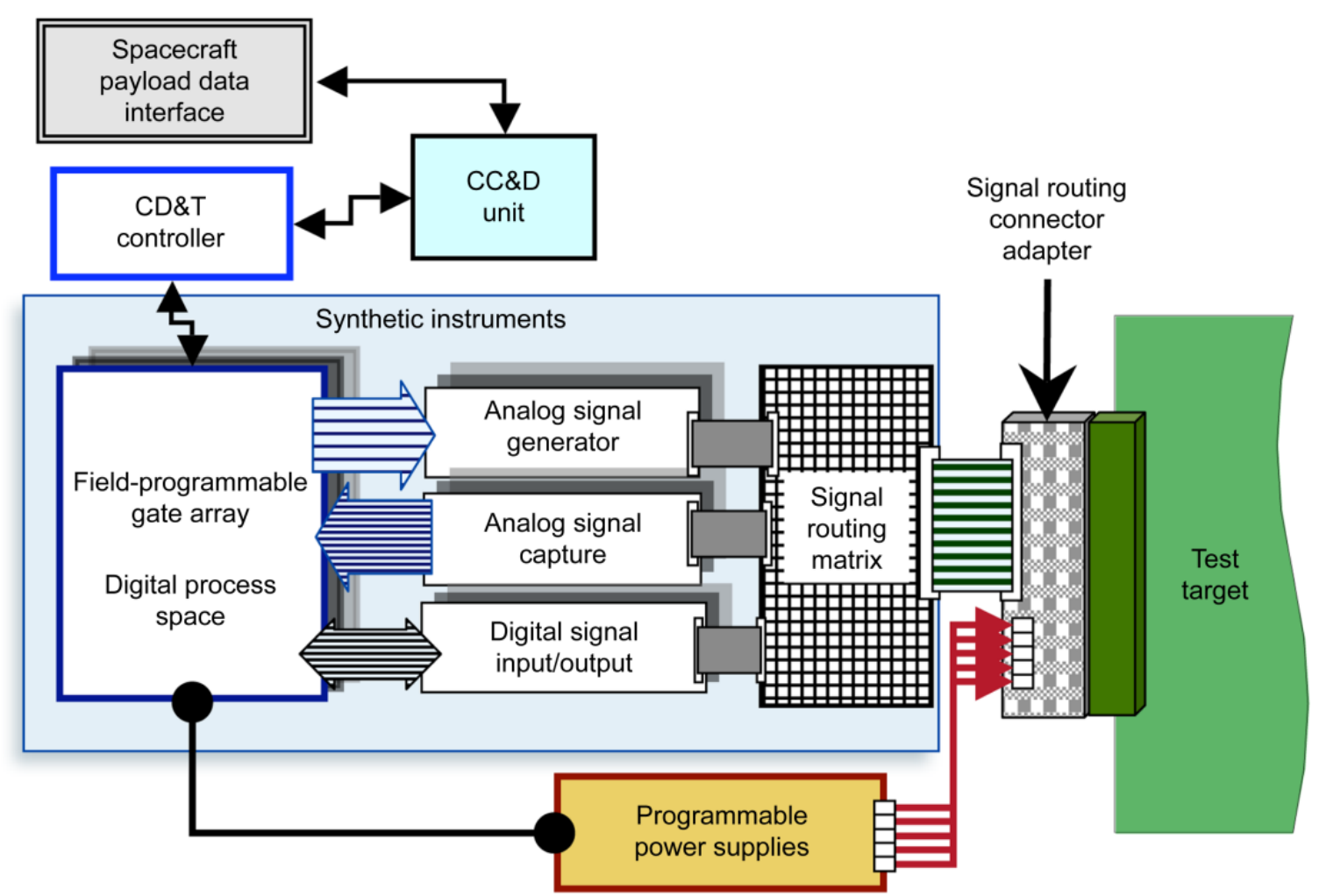

Figure 11.-A synthetic instrument (SI) architecture as a solution to diagnostic and test needs for future spacecraft repair capabilities.

For CLEAR, the vector network analyzer provides a complex impedance measurement. Network analyzers make their measurements relative to a reference impedance value (typically $50 \Omega$ for RF systems). As shown in Equation (1), complex impedance $Z$ is a complex value; that is, it is composed of a real value $R$ and an imaginary value $X$.

$$
Z=R+X i
$$

The $(R)$ value is the "resistance" term and the $(X)$ value is the "reactance" term and indicates the presence of inductance or capacitance in the circuit. Based on complex variable mathematics these terms can be combined into a single Complex Impedance value that can be physically measured by the vector network analyzer.

Complex impedance measured over a frequency sweep reveals resonant behaviors for both the entire network and individual component resonance. By performing a wide sweep, one can identify specific components. Despite the complexity of the underlying theory the indentifying a fault by graphical signature comparison is relatively easy and by comparing the test signature against a "gold" signature, one can quickly spot a failed component.

Figure 12 shows the concept of CSA in which a filter composed of several LC (inductor capacitor) nodes has a distinct resonant condition for each node. The plots produced by the network analyzer could be used to characterize the network. The actual measurement is taken only at the input end. Note that markers 1 through 5 indicate distinct nodes and each node's contribution to the characterization curve. Therefore, it is possible to characterize the network without measuring internal nodes. This is particularly useful when parts of a circuit are inaccessible. The measurement is taken from the signal injection side of the circuit rather than from the output side. In Figure 12, the blue trace is the characterization of a knowngood reference network. The red trace is a test trace from a network where node 2 has a faulty component. 

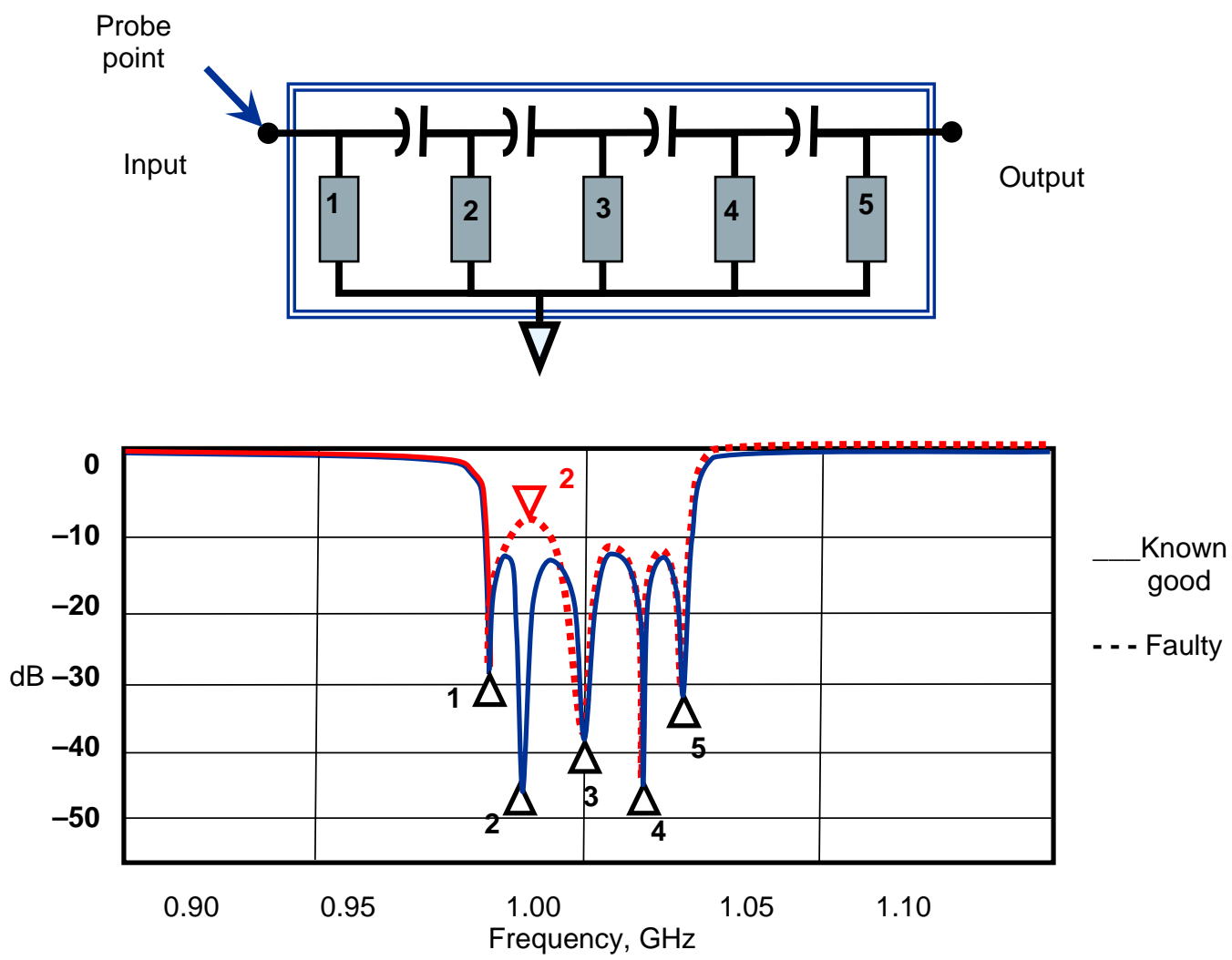

Figure 12.-Complex Signature Analysis (CSA)—Each marker indicates a normal resonance of a "known-good" network composed of five components. The red trace indicates that element 2 of the test circuit has a faulty component and therefore does not match up with the expected known-good trace.

In summary, ASA and CSA have similar approaches but measure distinctly different circuit behaviors. ASA operates in the time domain, whereas CSA operates in the frequency domain. ASA measures the response to a small voltage input like a component tester, whereas CSA measures complex impedance, which is a multi-component value that can be analyzed in different ways. The techniques have an overlapping range, but one technique does not encompass the capability of the other technique. Therefore, ASA and CSA diagnostics are considered to be complementary techniques. The overall Diagnostics Signature Analysis technology development will involve the combination of ASA and CSA techniques. CLEAR may further refine the capability by developing DSA as a Synthetic Instrument.

\subsubsection{Noncontact Diagnostics}

The CLEAR team conducted a preliminary feasibility study of noncontact techniques for circuit diagnostics. These techniques are intended to address the problems of node access in circuits that are protected by conformal coatings. Both ASA and CSA operate by injecting AC signals into the test circuit and examining the response. If a probe can be coupled by capacitive, inductive, or RF coupling, it may be feasible to inject signals without removing the conformal coating. The capacitive techniques used realistic physical parameters (size and materials) for the probes. The RF technique used the same coupling capacitance but higher frequencies. Inductive techniques were not pursued because the ability to model realistic injection of signals into the card being tested was limited.

The Simulation Program with Integrated Circuit Emphasis (SPICE) was used to simulate the test card and faults. For capacitive coupling, a signal was injected relative to a common ground on the circuit being 
tested. The results of the capacitive coupling techniques indicated that the test circuit's impedance dominated the response. The largest effective coupling capacitance that could realistically be achieved was less than $0.5 \mathrm{pF}$. Essentially, this technique is applicable for testing active components and boards operating at high frequency, but it is not applicable for low-frequency circuits. The frequency limitation of the test method could be removed by allowing differential measurement with dual-probe configurations. Although these techniques show promise, additional work will be required to refine the methods.

Simulations of capacitive probes show that this technique can be used for high-frequency components, such as antennas. However, when there are large inductors or capacitors on the board, the common ASA technique seems to be the best option. Unlike capacitive coupling probes, inductive probes do not seem to be practical for this application. The size of the inductive probe can be the major limiting factor. For inductive probes to be applicable, a board should be designed specifically for receiving these probes. Inductive coupling techniques have a strong dependency on the geometry of the board and the components close to the trace to be tested. Other electronic coupling techniques such as modulated RF coupling should be investigated.

In noncontact analysis, variations in the thickness of the coating layer can cause the analysis results to vary significantly. However, collecting reference information from each board for comparison to the same board in the future would eliminate the effect of coating thickness variation. This concept would allow relative comparison analysis to be applied instead of absolute analysis.

\subsection{Summary}

NASA's historical solution to the problem of in-flight electronics repair has been the replacement of sub-units called Orbital Replacement Units. This approach requires significant logistical support through regular resupply flights. For long-duration space missions, resupply flights are limited. Therefore, NASA is investigating component-level repair of electronics as a potential way to reduce the logistical footprint required to support future missions. The CLEAR task under the NASA Exploration Technology Developments Program's Supportability project was established to explore how future crews could effectively perform electronic repair down to the component level.

To better understand what types of electronic components would need to be repaired, the CLEAR team investigated electronic systems to determine the nature of the circuits used during spaceflight. Component-level repair will involve integrated circuit packaging that is expected to migrate from perimeter-mounted through-hole and surface-mount technologies to ball grid and land grid array technologies. This trend limits manual solder repair methods to simple discrete devices. High-density ball grid and land grid array devices must be repaired using automated techniques. This information was used as the basis to develop an architecture that addresses electronic diagnostics, repair, and testing needs, and the practical constraints of operating in a space environment.

The CLEAR System Architecture is comprised of an Earth and a space segment. The Earth segment is referred to as CLEAR Tele-operations Engineering Support Team which is comprised of system and operations experts. The ground-based CLEAR TEST center will be similar in concept to the various science payload operations centers available today. The space segment is referred to as the semiautomated repair and diagnostics apparatus (SARADA). The CLEAR System Architecture can allow flight crews, within the constraints of a spacecraft or habitat, to:

- Diagnose electronic assemblies and identify the faulty components

- Repair electronics down to the component-level

- Evaluate the circuit post repair via a functional test, and

- Augment the flight crew with knowledge and skills to diagnose faults and perform repairs without expanding crew size by a combination of manual and semi-automated processes 
In addition, this paper shows results from an analysis that component level repair offers both cost benefits and mass savings for extended space missions. Also, this paper discusses recent progress toward developing in-space repair capabilities including two space-flight experiments called SoRGE and CRE-1. Finally, this paper presents technology concepts which could help enable or benefit component-level repair. These concepts include developing synthetic instrumentation to meet in-situ testing needs, extending Analog Signature Analysis to the frequency domain using an approach called Complex Signature Analysis, and finally non-contact diagnostic methods to help diagnose faults without needing to penetrate conformal coating.

The proposed approach can increase the flight crew's ability to act decisively to resolve problems while reducing dependency on Earth-supplied logistics for future Constellation Program missions as well as the ISS.

\section{References}

1. Beddingfield, K.L., Leach, R.D., and Alexander, M.B. (eds.), "Spacecraft System Failures and Anomalies Attributed to the Natural Space Environment," NASA Reference Publication 1390, Aug. 1996.

2. Rutledge, P.J., Mosleh, A., "Dependent-Failures in Spacecraft: Root Causes, Coupling Factors, Defenses, and Design Implications,” Proceedings of the IEEE Annual Reliability and Maintainability Symposium, 0149-144X/95, (1995), pp. 337-342.

3. Columbia Accident Investigation Board, “Columbia Accident Investigation Board Report” [online report], Vol. 1, Aug. 2003 URL: http://caib.nasa.gov/news/report/volume1/default.html [cited 26 Dec. 2007].

4. Pettegrew, R.D., Easton, J.W., and Struk, P.M., "Repair of Electronics for Long Duration Spaceflight," $45^{\text {th }}$ AIAA Aerospace Sciences Meeting and Exhibit, Reno, NV, Jan. 8-11, 2007, AIAA-2007-1364.

5. Easton, J.W., Pettegrew, R.D., and Struk, P.M., "Electronic Repair Concepts for Long-Duration Spaceflight," $45^{\text {th }}$ AIAA Aerospace Sciences Meeting and Exhibit, Reno, NV, Jan. 8-11, 2007, AIAA-2007-545.

6. Pettegrew, R.D., Easton, J.W., Struk, P.M., and Anderson, E.E., "In-Flight Manual Electronics Repair for Deep-Space Missions,” Aerospace Conference, 2007 Institute of Electrical and Electronics Engineers (IEEE), Mar. 3-10, 2007.

7. Struk, P.M., Oeftering, R.C. Easton, J.W., and Anderson, E.E., "Semi-Automated Diagnosis, Repair, and Rework of Spacecraft Electronics," 46th AIAA Aerospace Sciences Meeting and Exhibit, Reno, NV, Jan. 7-10, 2008, AIAA-2008-1130.

8. Mankins, J.C., “Technology Readiness Levels: A White Paper,” Apr. 6, 1995, Advanced Concepts Office, Office of Space Access and Technology, URL: [cited 26 Dec. 2007].

9. Tate, J.M., Bartlett, J.K., Robinson, J.A., Maender, C.C., Putcha, L., Smith, S.M., Bowman, M.A., Dulchavsky, S.A., Sargsyan, A.E., Miller, S.K., Banks, B.A., deGroh, K.K., and Tsui, D., "Synergies between Space Research and Space Operations-Examples from the International Space Station," 45th AIAA Aerospace Sciences Meeting and Exhibit, Jan. 8-11, 2007, Reno, NV, AIAA-2007-547.

10. Grugel, R.N., Cotton, L.J., Segre, P.N., Ogle, J.A., Funkhouser, G., Parris, F., Murphy, L., Gillies, D., Hua, F., and Anilkumar, A.V., "The In-Space Soldering Investigation (ISSI): Melting and Solidification Experiment Aboard the International Space Station," $44^{\text {th }}$ AIAA Aerospace Sciences Meeting and Exhibit, Reno, NV, Jan. 8-12, 2006, AIAA-2006-521.

11. Easton, J.W., Struk, P.M., and Rotella, A., "Imaging and Analysis of Void Defects in Solder Joints Formed in Reduced Gravity Using High-Resolution Computed Tomography," $46^{\text {th }}$ AIAA Aerospace Sciences Meeting and Exhibit, Reno, NV, Jan. 7-10, 2008, AIAA-2008-0824.

12. Easton, J.W. and Struk, P.M., "Current Space Station Experiments Investigating Component Level Electronics Repair,” AIAA SPACE 2009 Conference \& Exposition, Sep. 14-17, 2009, Pasadena, CA, AIAA-2009-6798. 
13. Watson, J.K., Struk, P.M., Pettegrew, R.D., and Downs, R.S., Experimental Investigation of Solder Joint Defect Formation and Mitigation in Reduced-Gravity Environments, AIAA Journal of Spacecraft and Rockets, Vol. 44, No. 1 (Jan.-Feb. 2007), pp. 174-182.

14. Pettegrew, R.D., Struk, P.M., Watson, J.K., Haylett, D.R., and Downs, R.S. Gravitational Effects on Solder Joints, Welding Journal, 2003, pp. 44-48.

15. Struk, P.M., Pettegrew R.D., Downs, R.S., and Watson, J.K., The Influence of Gravity on Joint Shape for Through-Hole Soldering, $43^{\text {rd }}$ AIAA Aerospace Sciences Meeting and Exhibit, Reno, NV, Jan. 10-13, 2005, AIAA-2005-0541 and NASA/TM-2005-213589, 2005.

16. Struk, P.M., Pettegrew, R.D., Downs, R.S., and Watson, J.K., The Effects of an Unsteady Reduced Gravity Environment on the Soldering Process, $42^{\text {nd }}$ AIAA Aerospace Sciences Meeting and Exhibit, Reno, NV, Jan. 5-8, 2004, AIAA-2004-1311 and NASA/TM-2004-212946, 2004.

17. Pettegrew, R.D., Struk, P.M., Watson, J.K., and Haylett, D.R., "Experimental Methods in Reduced Gravity Research,” NASA/TM-2002-211993, 2002.

18. Easton, J.W., Struk, P.M., Funk, G.P., Latta, G.S., Ganster, A.W., Estes, B.E., "Recommendations for Enabling Manual Component Level Electronic Repair for Future Space Missions,” NASA Technical Memorandum, in-preparation.

19. Humprey, R., Addressing future test challenges via synthetic instrumentation, Military Embedded Systems, May 2006, URL: http://www.mil-embedded.com/articles/authors/humphrey/ [cited 26 Dec. 2007]. 


\begin{tabular}{|c|c|c|}
\hline \multicolumn{2}{|c|}{ REPORT DOCUMENTATION PAGE } & $\begin{array}{l}\text { Form Approved } \\
\text { OMB No. 0704-0188 }\end{array}$ \\
\hline \multicolumn{3}{|c|}{ 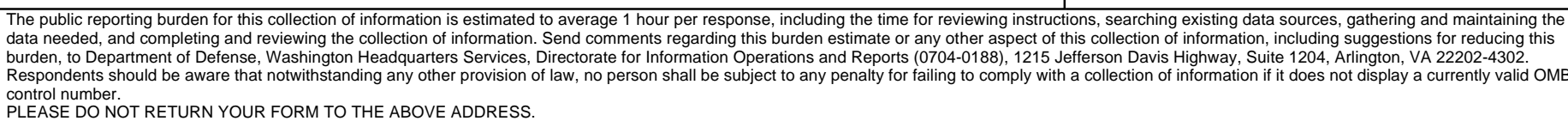 } \\
\hline $\begin{array}{l}\text { 1. REPORT DATE (DD-MM-YYYY) } \\
01-09-2010\end{array}$ & $\begin{array}{l}\text { 2. REPORT TYPE } \\
\text { Technical Memorandum }\end{array}$ & 3. DATES COVERED (From - To) \\
\hline \multirow{3}{*}{\multicolumn{2}{|c|}{$\begin{array}{l}\text { 4. TITLE AND SUBTITLE } \\
\text { Approach to In Situ Component Level Electronics Assembly Repair (CLEAR) for } \\
\text { Constellation }\end{array}$}} & 5a. CONTRACT NUMBER \\
\hline & & 5b. GRANT NUMBER \\
\hline & & 5c. PROGRAM ELEMENT NUMBER \\
\hline \multirow{3}{*}{\multicolumn{2}{|c|}{$\begin{array}{l}\text { 6. AUTHOR(S) } \\
\text { Struk, Peter, M.; Oeftering, Richard, C. }\end{array}$}} & 5d. PROJECT NUMBER \\
\hline & & 5e. TASK NUMBER \\
\hline & & $\begin{array}{l}\text { 5f. WORK UNIT NUMBER } \\
\text { WBS 825855.01.03.03.03 }\end{array}$ \\
\hline \multicolumn{2}{|c|}{$\begin{array}{l}\text { 7. PERFORMING ORGANIZATION NAME(S) AND ADDRESS(ES) } \\
\text { National Aeronautics and Space Administration } \\
\text { John H. Glenn Research Center at Lewis Field } \\
\text { Cleveland, Ohio 44135-3191 }\end{array}$} & $\begin{array}{l}\text { 8. PERFORMING ORGANIZATION } \\
\text { REPORT NUMBER } \\
\text { E-17412 }\end{array}$ \\
\hline \multirow{2}{*}{\multicolumn{2}{|c|}{$\begin{array}{l}\text { 9. SPONSORING/MONITORING AGENCY NAME(S) AND ADDRESS(ES) } \\
\text { National Aeronautics and Space Administration } \\
\text { Washington, DC 20546-0001 }\end{array}$}} & $\begin{array}{l}\text { 10. SPONSORING/MONITOR'S } \\
\text { ACRONYM(S) } \\
\text { NASA }\end{array}$ \\
\hline & & $\begin{array}{l}\text { 11. SPONSORING/MONITORING } \\
\text { REPORT NUMBER } \\
\text { NASA/TM-2010-216784 }\end{array}$ \\
\hline \multicolumn{3}{|c|}{$\begin{array}{l}\text { 12. DISTRIBUTION/AVAILABILITY STATEMENT } \\
\text { Unclassified-Unlimited } \\
\text { Subject Categories: } 12,16,18 \text {, and } 33 \\
\text { Available electronically at http://gltrs.grc.nasa.gov } \\
\text { This publication is available from the NASA Center for AeroSpace Information, 443-757-5802 }\end{array}$} \\
\hline
\end{tabular}

\section{SUPPLEMENTARY NOTES}

\section{ABSTRACT}

Maintenance resupply is a significant issue for long duration space missions. Currently, the International Space Station (ISS) approaches maintenance primarily around replaceable modules called Orbital Replacement Units (ORU). While swapping out ORUs has served the ISS well keeping crew time for maintenance to a minimum, this approach assumes a substantial logistics capacity to provide replacement ORUs and return ORUs to Earth for repair. The ORUs used for ISS require relatively large blocks of replacement hardware even though the actual failed component may be several orders of magnitude smaller. The Component Level Electronics Assembly Repair (CLEAR) task was created to explore electronics repair down to the component level for future space missions. From 2006 to 2009, CLEAR was an activity under the Supportability project of the Exploration Technology Development Program. This paper describes the activities of CLEAR including making a case for component-level electronics repair, examination of current terrestrial repair hardware, and potential repair needs. Based on those needs, the CLEAR team proposes an architecture for an in-situ repair capability aboard a spacecraft or habitat. Additionally, this paper discusses recent progress toward developing in-space repair capabilities--including two spaceflight experiments-and presents technology concepts which could help enable or benefit the same.

15. SUBJECT TERMS

Electronics; Maintenance; Soldering; Diagnostics; Space maintenance; Telerobotics

\begin{tabular}{|l|l|l|l|c|l|}
\hline \multicolumn{2}{|l|}{ 16. SECURITY CLASSIFICATION OF: } & $\begin{array}{l}\text { 17. LIMITATION OF } \\
\text { ABSTRACT }\end{array}$ & $\begin{array}{l}\text { 18. NUMBER } \\
\text { OF } \\
\text { PAGES }\end{array}$ & $\begin{array}{l}\text { 19a. NAME OF RESPONSIBLE PERSON } \\
\text { STI Help Desk (email:help@sti.nasa.gov) }\end{array}$ \\
\cline { 1 - 1 } $\begin{array}{l}\text { a. REPORT } \\
\text { U }\end{array}$ & $\begin{array}{l}\text { b. ABSTRACT } \\
\text { U }\end{array}$ & $\begin{array}{l}\text { c. THIS } \\
\text { PAGE } \\
\text { U }\end{array}$ & UU & 37 & $\begin{array}{l}\text { 19b. TELEPHONE NUMBER (include area code) } \\
\text { 4t43-757-5802 }\end{array}$ \\
\hline
\end{tabular}



